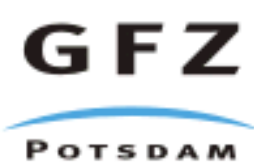

Originally published as:

Wicht, J., Mandea, M., Takahashi, F., Christensen, U. R., Matsushima, M., Langlais, B. (2007): The origin of Mercury's internal magnetic field. - Space Science Reviews, 132, 2-4, 261-290,

DOI: $10.1007 / s 11214-007-9280-5$. 


\title{
The Origin of Mercury's Internal Magnetic Field
}

\author{
J. Wicht • M. Mandea • F. Takahashi · U.R. Christensen • \\ M. Matsushima $\cdot$ B. Langlais
}

Received: 23 January 2007 / Accepted: 14 September 2007

(C) Springer Science+Business Media B.V. 2007

\begin{abstract}
Mariner 10 measurements proved the existence of a large-scale internal magnetic field on Mercury. The observed field amplitude, however, is too weak to be compatible with typical convective planetary dynamos. The Lorentz force based on an extrapolation of Mariner 10 data to the dynamo region is $10^{-4}$ times smaller than the Coriolis force. This is at odds with the idea that planetary dynamos are thought to work in the so-called magnetostrophic regime, where Coriolis force and Lorentz force should be of comparable magnitude. Recent convective dynamo simulations reviewed here seem to resolve this caveat. We show that the available convective power indeed suffices to drive a magnetostrophic dynamo even when the heat flow though Mercury's core-mantle boundary is subadiabatic, as suggested by thermal evolution models. Two possible causes are analyzed that could explain why the observations do not reflect a stronger internal field. First, toroidal magnetic fields can be strong but are confined to the conductive core, and second, the observations do not resolve potentially strong small-scale contributions. We review different dynamo simulations that promote either or both effects by (1) strongly driving convection, (2) assuming a particularly small inner core, or (3) assuming a very large inner core. These models still fall somewhat short of explaining the low amplitude of Mariner 10 observations, but the incorporation of an additional effect helps to reach this goal: The subadiabatic heat flow through Mercury's core-mantle boundary may cause the outer part of the core to be stably stratified, which would largely exclude convective motions in this region. The magnetic field, which is
\end{abstract}

J. Wicht $(\bowtie) \cdot$ U.R. Christensen

Max-Planck Institute for Solar-System Research, 37191 Kaltenburg-Lindau, Germany

e-mail: wicht@mps.mpg.de

M. Mandea

GeoForschungsZentrum Potsdam, Telegrafenberg, 14473 Potsdam, Germany

F. Takahashi · M. Matsushima

Department of Earth and Planetary Sciences, Tokyo Institute of Technology, 2-12-1 Ookayama,

Meguro-ku, Tokyo 152-8551, Japan

B. Langlais

UMR CNRS 6112, Laboratoire de Planétologie et Géodynamique, Nantes Atlantique Universités, 2 Rue de la Houssinière, 44000 Nantes, France 
small scale, strong, and very time dependent in the lower convective part of the core, must diffuse through the stagnant layer. Here, the electromagnetic skin effect filters out the more rapidly varying high-order contributions and mainly leaves behind the weaker and slower varying dipole and quadrupole components (Christensen in Nature 444:1056-1058, 2006). Messenger and BepiColombo data will allow us to discriminate between the various models in terms of the magnetic fields spatial structure, its degree of axisymmetry, and its secular variation.

Keywords Mercury: magnetic field · Mercury: dynamo

\section{Introduction}

Our knowledge of Mercury's internal magnetic field is scarce. Data from two close flybys by Mariner 10 in 1974 and 1975, half of the first flyby being rendered useless due to strong magnetospheric perturbations, is all we have. These data give only limited information about the internal field structure (Connerney and Ness 1988), but the existence of an internal field was a surprise in itself.

Prior to the Mariner 10 encounters, it was thought that Mercury had no active dynamo. If the planet ever had a dynamo it would have ceased to work when the rapid cooling of the comparatively small planet led to a completely frozen core. This idea was disproved by the Mariner 10 measurements, suggesting that at least part of the metal core must still be liquid for a convective dynamo to operate. Recent ground-based observations of Mercury's forced libration are also in favor of a partly liquid core, since their large amplitude requires a mechanical decoupling of core and mantle (Margot et al. 2007). The low observed field strength, however, seems incommensurate with typical convective dynamos. The Earth's magnetic field, for example, is two orders of magnitude stronger.

This motivated scientists to look for alternative models. Stevenson (1987) and later Giampieri and Balogh (2002) suggested a thermoelectric mechanism: Temperature differences at the core-mantle boundary caused by topographic undulations would give rise to a thermoelectric electro-motive force that drives electric currents in core and mantle. These currents are the source for a toroidal magnetic field that in turn is converted into the observed poloidal field by convective flows too sluggish to support a classical self-sustained dynamo. The parameterized models by Giampieri and Balogh (2002) show that the resulting field strength is compatible with the observations. The field structure is determined by the undulation of the core-mantle boundary and the structure of the convective flow. A significant correlation between gravity and magnetic field pattern detected by future Mercury missions would be in favor of the thermoelectric dynamo model (Giampieri and Balogh 2002), assuming that the gravity signal reflects the core-mantle boundary shape.

Remanent crustal magnetization has also been suggested as a possible source for the global magnetic field observed by Mariner 10. Since Mars Global Surveyor discovered a surprisingly strong magnetization of the Martian crust (Acuña et al. 1999), the Hermian field amplitude seems within reach of remanent magnetization models (Aharonson et al. 2004). However, the magnetization of the Martian and Earth's crust is rather complex and small scale (see Sects. 2.1 and 2.2). The pattern found on Earth is a result of long-term tectonic and magnetic processes conserving the varying direction of a reversing magnetic field. Variations in the magnetic susceptibility of lithospheric rocks are responsible for additional smaller modulations. The reason for the complexity of Martian magnetization is less clear, but similar mechanisms are typically evoked. 
A simple magnetization pattern compliant with Mariner 10 observations is possible only if Earth-like tectonic processes are absent and the dynamo is not reversing during the acquisition of magnetization. Mercury's surface is predominantly shaped by impact processes, so that the first condition is fulfilled. The latter condition also seems little restrictive, given the fact that many published dynamo simulations never reverse. However, we will see in the following that Mercury's dynamo may operate in a regime where magnetic field reversals are expected. A theorem by Runcorn (1975) states that a uniform spherical shell magnetized by an internal source that is subsequently removed has no external field. Having ruled out tectonics, one therefore has to invoke a different mechanism that breaks the uniformity on a larger scale. Aharonson et al. (2004), for example, suggested that the strong latitudinal and longitudinal variations in Mercury's mean surface temperature lead to a varying thickness of the magnetized crustal layer because the Curie temperature is reached at different depths. A key feature that will allow us to identify crustal magnetization-once MESSENGER and BepiColombo provide new data - is the correlation of magnetic field features with topographic entities like craters (Aharonson et al. 2004).

The weakness of Mercury's internal magnetic field has the consequence that external and internal contributions are harder to separate than in the geomagnetic field, where they differ by orders of magnitude. The external field can reach amplitudes comparable to the dynamo field strength above the planet's surface (Fujimoto et al. 2007). So far, external field models were simply scaled down versions of Earth's magnetospheric dynamics. This, however, may fall short in the Mercury case. Field contributions due to electric currents induced in the core by external field variations and due to convective alteration of the external field also have to be considered (Glassmeier et al. 2007). Exploring the tight link of internal and external sources may require us to develop a dynamo model that incorporates the interaction with the external field.

After giving an overview of different magnetic fields of terrestrial planets in Sect. 2, we concentrate on convective dynamo simulations for the remainder of the article. Numerical simulations geared to model the geodynamo successfully reproduce the geomagnetic field strength and are thus not directly suited to explain the weaker Hermian field (Christensen and Aubert 2006). Typical estimates for the field strength of convectively driven planetary dynamos likewise predict much stronger fields than observed by Mariner 10 (Stevenson 1987). We review this issue in Sect. 4 where we also discuss the power available to drive Mercury's dynamo. Although some researchers challenged the applicability of a convective dynamo model (Stevenson 1987; Schubert et al. 1988), more recent numerical simulations have resolved the caveat by exploring the influence of possible differences in Earth's and Mercury's interior on the dynamo process. Smaller as well as larger inner cores are assumed, and both can help to reduce the global field strength (at satellite altitude) compared to an Earth-like relative inner-core size. We present both types of models in Sect. 5 after having introduced the numerical dynamo problem in Sect. 3. Simulations of Mercury's thermal evolution suggest that the inner core still grows while the core-mantle boundary temperature gradient is subadiabatic. The convection, which is chemically driven in the deeper regions of the fluid shell, could therefore be stably stratified due to thermal effects in the outer part of the core. We present dynamo models that assume this mixed type of convection in Sect. 6. A discussion in Sect. 7 concludes this article and assesses how future Messenger and BepiColombo measurements could distinguish between the different models. 


\section{Earth-Like Planetary Magnetic Fields}

Nowadays, measurements of planetary magnetic fields are mainly devoted to understanding the planet's interior structure and dynamics as well as the near-planetary environment. The existence of an interior field is a strong indicator that at least part of the iron core must still be liquid and convects vigorously enough to drive a dynamo. Variations in the internal magnetic field bear valuable, and often the only available, information on the core dynamics.

The planetary dynamos in our solar system seem to be quite diverse. Jupiter's and Earth's magnetic fields are both dominated by the axial dipole contribution but differ significantly in strength. The dipole plays no special role in the fields of Uranus and Neptune and is also significantly tilted. Saturn's magnetic field, on the other hand, seems to be extremely axisymmetric and somewhat weak. Venus and Mars have no active dynamos at present, and Mercury's field seems to be large scale but is exceptionally weak. Explaining these differences is an interesting challenge for planetary research in general and dynamo modeling in particular.

To get an understanding of the variability of terrestrial dynamos, we outline the key features of the magnetic fields of Earth, Mars, Venus, and Mercury, and also add Earth's Moon for good measure. Planetary fields may contain contributions from different external and internal sources. The interaction between the internal magnetic field and the solar wind results in a current system that is responsible for external magnetic fields (Fujimoto et al. 2007). Magnetized crust is an additional potential magnetic field source. Separating and understanding the different contributions is a formidable task that requires as many data in space and time as possible.

Naturally, the data coverage is by far the best for our home planet. More than 200 years of continuous observations of the vector magnetic field and measurements from several spacecrafts providing global geographical coverage have established a good, but still incomplete, knowledge of the field morphology and its time evolution. The case is different for the other planets, where spacecraft are the only means of measuring the magnetic field. Sometimes, as in the case of Mars Global Surveyor, we are lucky and the spacecraft orbits a planet for several years. In other cases our knowledge is based on only one flyby.

\subsection{The Earth}

The Earth's magnetic field is the sum of several internal and external contributions: Crustal and dynamo fields are of internal origin, ionospheric and magnetospheric fields form the external part. Various data sets from different sources are available to describe and separate these contributions and range from historic magnetic measurements to recent high-quality data provided by magnetic observatories (worldwide but unevenly distributed) and the magnetic satellites MAGSAT, SAC-C, Ørsted, and CHAMP.

Figure 1 shows the total intensity of the dynamo field at Earth's surface, which is the by far dominant field contribution. It reaches magnitudes of approximately 60,000 nT (nano tesla) near the poles, and decreases to values less than 25,000 nT in the South-Atlantic Anomaly region. Time variations of the core field, the secular variation, mainly occur on decades and longer time scales, but geomagnetic data have also revealed more abrupt changes in the trend of the secular variation, that last about one year and have been called geomagnetic jerks or secular variation impulses (Mandea et al. 2000).

The lithospheric field, shown in Fig. 2, is considered to be constant in time in comparison and originates from the crust and upper mantle. Crustal magnetization, both remanent and induced, may reach amplitudes of some 1,000 nT and cover large areas. The Kursk anomaly 


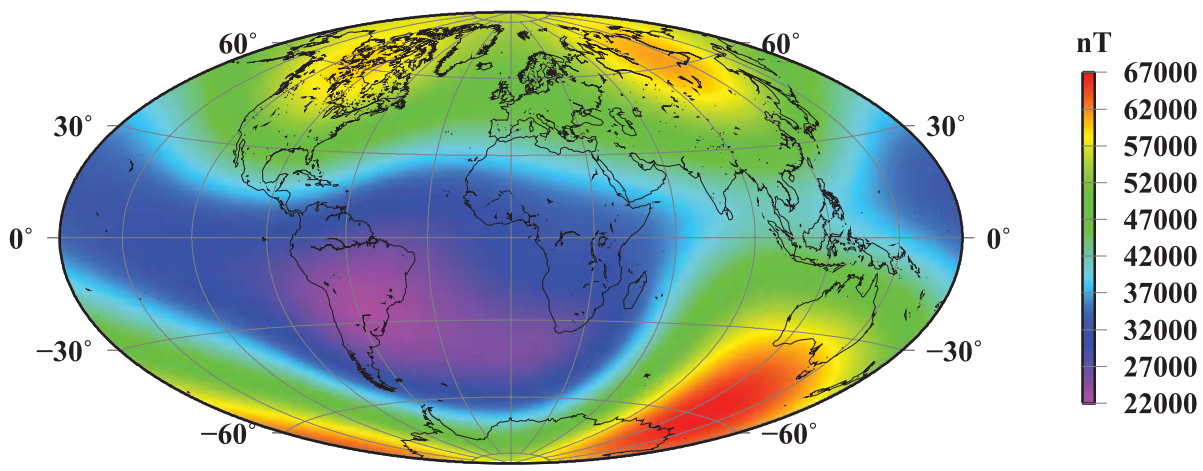

Fig. 1 The geomagnetic field intensity at the Earth's surface for epoch 2005.0 as given by the CHAOS model (Olsen et al. 2006)

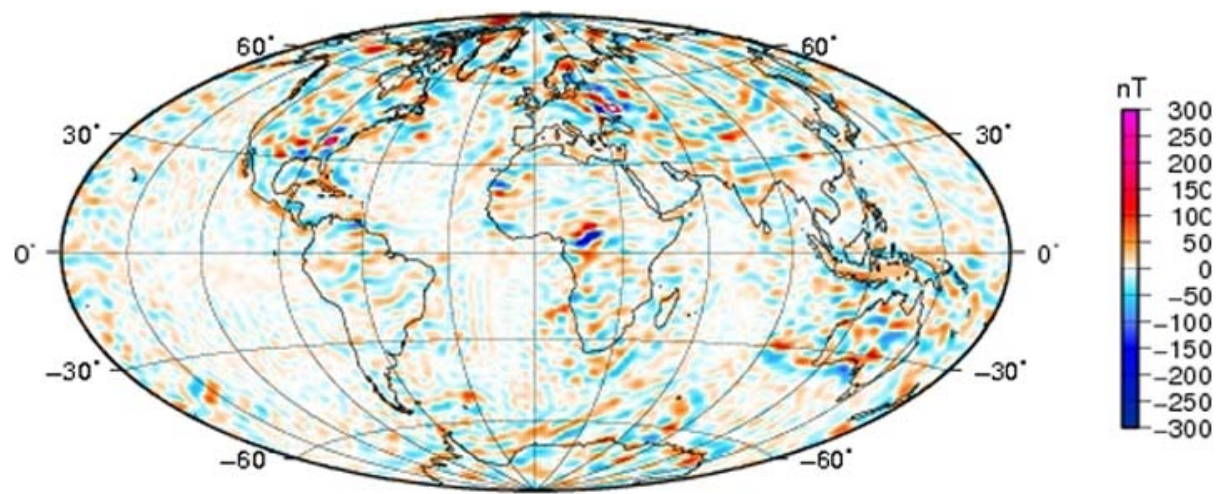

Fig. 2 Total intensity field based on the lithospheric field model MFX4 (Lesur and Maus 2006)

in the Ukraine is a prominent example. Banded Iron Formations and other rock types peculiar to the Proterozoic era are common in surface exposures of these regions. In the ocean area, striped anomalies are associated with sea-floor spreading; this pattern is characterized by a nearly constant magnetization direction over many hundreds of $\mathrm{km}$ and can be correlated with the dipole reversal sequence over millions of years. More details on magnetic field contributions of internal origin can be found in Mandea and Purucker (2005).

At distances of more than two Earth radii, the geomagnetic field can be approximated by a centered dipole that is inclined by $11^{\circ}$ with respect to the planet's rotation axis. Closer to the planet, at altitudes between about 50 and $600 \mathrm{~km}$, lies the ionosphere. Ultraviolet light from the Sun ionizes atoms in the upper atmosphere, making the day side of the ionosphere significantly more electrically conducting than the night side. Strong electric currents circulating in the sunlit hemisphere generate magnetic fields that contribute with intensities of up to $80 \mathrm{nT}$ to the surface field. The respective daily cycle gives rise to the so called solar-quiet variations. Solar storms and other more energetic nonquiet events linked to the activity of the Sun may create magnetic fields up to a few thousand nT.

The Earth's magnetosphere, which extends for about 10 Earth radii in the direction towards the Sun and much further on the night side, hosts a complex system of electric currents (Fujimoto et al. 2007). It is dominated by the equatorial ring current (Glassmeier et al. 
2007) whose contribution closer to the planet can be described by a uniform field that continuously varies with periods of six months and one year. These variations induce electric currents in Earth's interior which in turn produce the induced magnetic field that depends on the electrical conductivity structure of the planetary body. Secondary induced fields are, for example, caused by the motion of electrically conducting seawater through the Earth's main magnetic field (Tyler et al. 1997). Though the respective fields amount to only a few nT in magnitude, the effects of the regular lunar semidiurnal tide could nevertheless be discerned in geomagnetic satellite observations (Tyler et al. 2003). Large-scale ocean currents, such as the Antarctic Circumpolar Current, are also expected to produce signatures of up to a few $\mathrm{nT}$ at satellite altitude.

\subsection{Mars}

Though Mars has no active dynamo, a highly irregular field of lithospheric origin was detected. The most likely scenario is that the crust acquired an intense remanent magnetization while cooling in the presence of a still-active strong dynamo early in the planet's history (Arkani-Hamed 2007). Recent magnetic field maps (Langlais et al. 2004; Connerney et al. 2005) are based on compiled measurement by the Mars Global Surveyor (see Fig. 3). Amplitudes of up to $250 \mathrm{nT}$ are reached at $400 \mathrm{~km}$ altitude. External contributions are weaker and result from the interaction between the solar wind and the thin Martian atmosphere. Most of these interactions take place on the day side and cause a highly variable dynamic magnetosphere both in intensity and in altitude.

Mars' magnetic field is about one order of magnitude more intense than the lithospheric component of the terrestrial field. No surface measurements are currently available, but model predictions suggest that it could locally reach up to $10,000 \mathrm{nT}$ or more (Langlais et al. 2004), which is equivalent to the core field at Earth's surface.

Figure 3 shows a map of the vertical component of the magnetic field. The Martian magnetization is highly inhomogeneous, the southern highlands being apparently more magnetized than the northern lowlands. The largest volcanic edifices (Tharsis, Elysium, Olympus) as well as the largest impact craters (Hellas, Argyre, Isidis) are devoid of significant magnetic field at satellite altitude, which is generally attributed to local demagnetization of an otherwise magnetized crust. Thermal demagnetization due to volcanoes

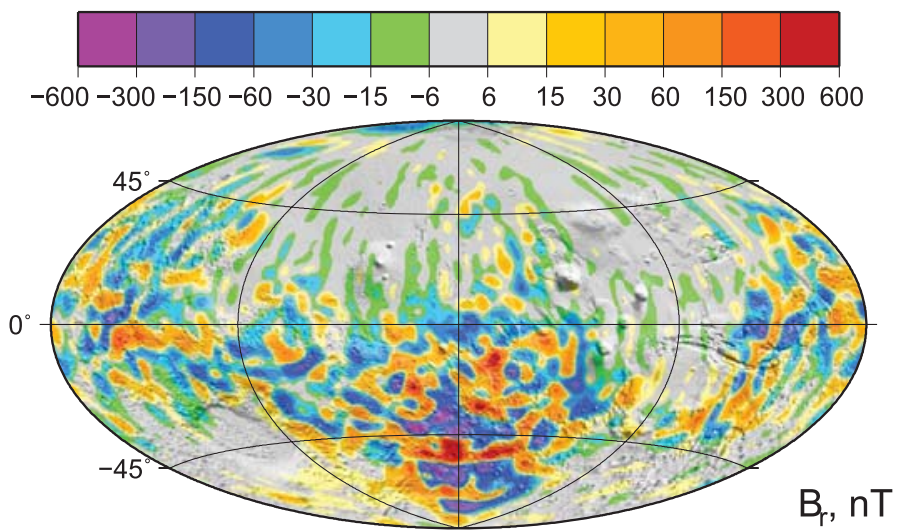

Fig. 3 Vertical component of the Martian field at $200 \mathrm{~km}$ altitude (Langlais et al. 2004) 
and impacts as well as impact excavations are likely explanations (Artemieva et al. 2005; Carporzen et al. 2005).

The magnetization identified in a Martian meteorite dated at $4 \mathrm{Ga}$ supports the idea of an early dynamo (Weiss et al. 2004) which was most likely driven by convection. Cooling of the mantle early in the planet's history and the associated increase in mantle viscosity severely lowered the vigor of mantle convection. This, in turn, reduced the heat flow though the coremantle boundary and eventually caused the dynamo to shut off (Breuer and Spohn 2003; Buske and Christensen 2007). Thermal evolution simulations suggest that the Martian core is still completely liquid, so that a chemically driven dynamo can be ruled out (Stevenson et al. 1983; Spohn et al. 2001; Williams and Nimmo 2004; Buske and Christensen 2007; see also Sect. 4).

\subsection{The Moon}

Early measurements showed that the Moon has no global magnetic field but possesses a magnetic field of lithospheric origin. The lunar magnetic field has been studied indirectly via the natural remanent magnetization of the returned lunar samples, and directly with magnetometers placed on the surface or in low-altitude orbits by the Apollo 15 and 16 missions. These measurements reveal widespread lunar magnetism on scales ranging up to many tens of kilometers. Paleointensity measurements on the returned samples suggest that the lunar surface field was comparable in intensity to the present-day terrestrial surface field about 3.6 to 3.8 Gy ago. Recent measurements by Lunar Prospector refined our understanding of the lunar magnetic field (Hood et al. 2001). Relatively weak fields (up to some tens of nT at spacecraft altitude) were mostly recorded antipodal to the largest lunar basins (Imbrium, Crisium, Serenitatis) (Anderson and Wilhelms 1979). This suggests that these magnetic anomalies are caused by the ejecta of impact processes, that were deposited at antipodal locations in the presence of an impact-amplified magnetic field (Hood et al. 2001).

\subsection{Venus}

Despite its relative similarity to the Earth in terms of size and density, Venus is devoid of any intrinsic magnetic field. Mariner 5 approached the planet to 1.4 Venus radii in 1967 and detected only signatures of solar wind deflection. The Pioneer Venus Orbiter mission measured the magnetic field during its first years of operation between 1979 and 1981. Lowaltitude observations $(\simeq 150 \mathrm{~km}$ ) on both the night and day sides proved that the observed field can be explained by the solar wind interaction with the planet alone; any internal field is insignificant at satellite altitude (Luhmann 1986).

\subsection{Mercury}

Mariner 10 recorded Mercury's magnetic field during the two close flybys Mariner I and Mariner III. Figure 4 displays the measurements in a body-centered Cartesian coordinate system; the $Z$-direction points northward and the $X$-direction points towards the Sun. Encounter I had its closest approach of $1.29 R_{M}$ at a latitude of $2^{\circ}$ south and recorded a maximum field strength of $98 \mathrm{nT}$. Encounter III had its closest approach at $1.13 R_{M}$ and a latitude of $68^{\circ}$ north and recorded a maximum field strength of $400 \mathrm{nT}$. The increased fluxes of energetic particles detected during the second half of Mariner I were attributed to a magnetospheric substorm (Siscoe et al. 1975), which also disturbed the magnetic recordings (see Fig. 4). Mariner I probably also led the spacecraft through the tail current sheet which 
Fig. 4 The Mercury magnetic field as observed during the first (blue) and third (red) flybys of Mariner 10
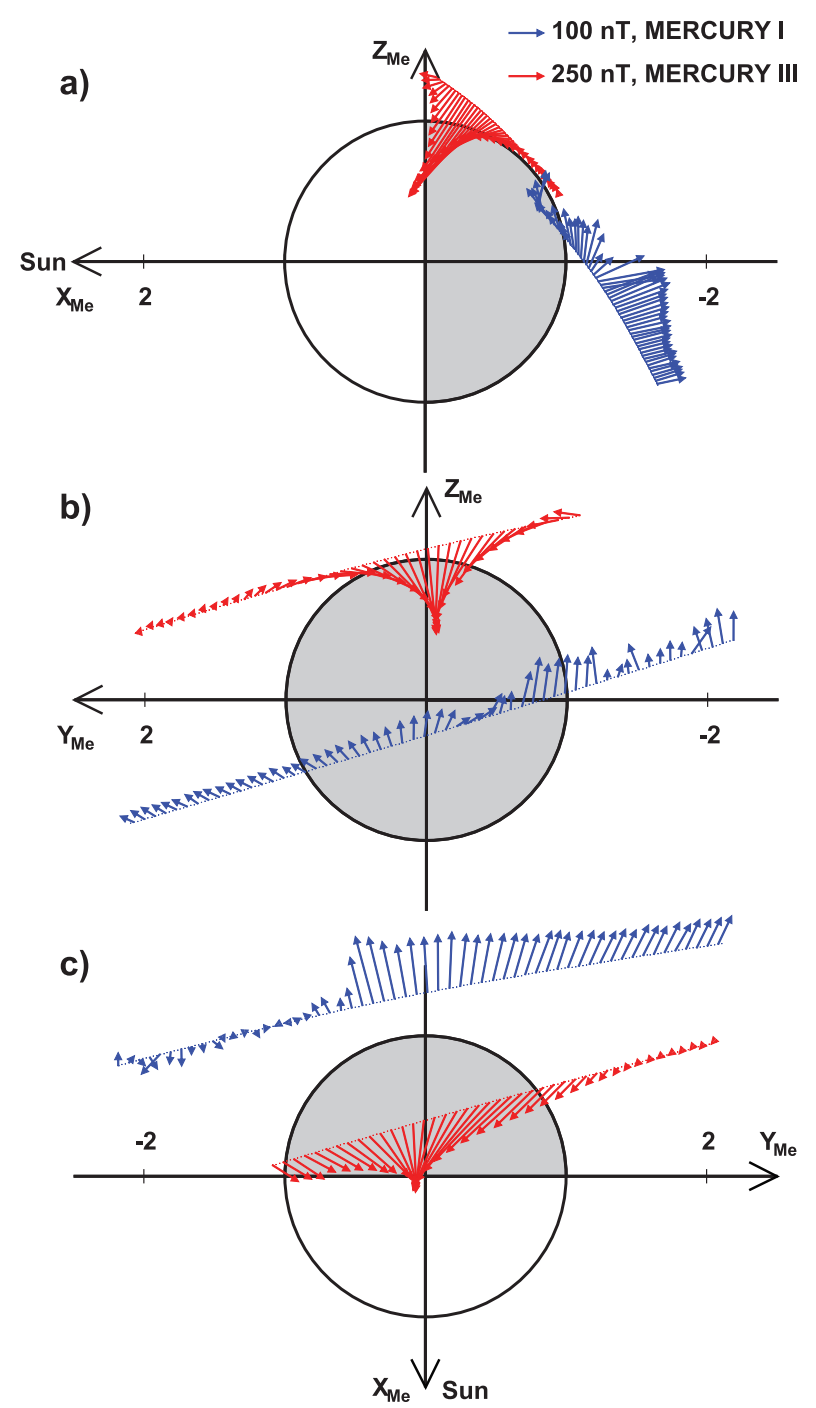

complicates the extraction of internal field contributions (Fujimoto et al. 2007). A higher latitude was therefore chosen for Mariner III to reduce these problems.

The data scarcity and uncertain external field contributions render Mercury's internal magnetic field models as highly ambiguous (Connerney and Ness 1988). External field contributions can reach the same order of magnitude as the internal field. Moreover, internal magnetic fields from induction due to external field variations and/or convective modification of the external field may not be neglected (Glassmeier et al. 2007). Commonly, the planetary surface field configuration is expressed in Schmidt normalized spherical harmonic coefficients $g_{l m}$ and $h_{l m}$ that denote the cosine and sine contributions of degree $l$ and order $m$, respectively. Most authors fitted axial dipole $g_{10}$ and axial quadrupole $g_{20}$ components to the data from the encounter tracks that lie inside a scaled-down Earth-like magnetosphere (Fujimoto et al. 2007). 
Estimates of the axial dipole component range from $g_{10}=-260 \mathrm{nT}$ to $g_{10}=-350 \mathrm{nT}$, those for the axial quadrupole components range from $g_{20}=-120 \mathrm{nT}$ to zero (see Connerney and Ness 1988, for an overview of Hermian field models). Connerney and Ness (1988) explored the ambiguities within a model family that represents the internal field by degree $l=1$ harmonics plus an axial quadrupole term and assumes a degree $l=1$ external field. They found that the model vector of least sensitivity to the specific flyby track III has the relative internal components:

$$
g_{10}=0.76, \quad g_{11}=0.23, \quad h_{11}=0.03, \quad g_{20}=-0.50 .
$$

This result can be interpreted such that a change in the dipole contribution of any internal field model by $\Delta g_{10}$ would lead to an equivalently valid model as long as the axial quadrupole is also changed by $\Delta g_{20}=-0.50 / 0.76 \Delta g_{10}=-0.66 \Delta g_{10}$. When taking into account that size and shape of the magnetosphere put additional constraints on the model coefficients, this ambiguity explains the range of internal field models found in the literature. The data do also allow for a significant harmonic order $m=1$ component, which could give rise to a dipole tilt that Ness (1979) estimated to $14 \pm 5^{\circ}$. Significant higher order contributions seem unlikely at satellite altitude given the fact that the magnetic field vector changed rather smoothly during encounter III. Because of their stronger decay with radius, these contributions could nevertheless contribute significantly in Mercury's dynamo region.

\section{Numerical Dynamo Models}

Self-consistent numerical dynamo models solve for convection and magnetic field generation in a rotating spherical shell filled with an electrically conducting fluid. Evolution equations for flow $\mathbf{u}$, magnetic field $\mathbf{B}$, and the codensity variable $b$ are formulated in a frame of reference corotating with the planetary mantle. The codensity combines dynamically relevant density differences due to thermal and compositional origin. The equations are made dimensionless by assuming scales that seem appropriate to the problem. We mostly follow the scaling chosen by Christensen et al. (2001), taking the shell thickness $D=r_{o}-r_{i}$ as length scale, the viscous diffusion time $D^{2} / v$ as time scale, $\left(\rho_{o} \mu \lambda \Omega\right)^{1 / 2}$ as the magnetic scale, and $\rho_{o}$ as the codensity scale.

The physical properties of the problem are $r_{i}$ and $r_{o}$, the inner and outer boundary radii respectively, mean outer-core density $\rho_{o}$, magnetic permeability $\mu$, magnetic conductivity $\sigma$, magnetic diffusivity $\lambda=1 /(\mu \sigma)$, planetary rotation rate $\Omega$, and kinematic viscosity $\nu$. These properties are condensed into five dimensionless parameters that govern the problem: Ekman number $E=v /\left(D^{2} \Omega\right)$, Prandtl number $P r=v / \kappa$, magnetic Prandtl number $P m=$ $\nu / \lambda$, Rayleigh number $R a=g_{o} \Delta b D /(\nu \Omega)$, and aspect ratio $\eta=r_{i} / r_{o}$. Here, $\kappa$ stands for a diffusivity that combines thermal and compositional effects (see the following). Gravity $g_{o}$ at the outer boundary is taken as the reference value, and we assume that gravity varies linearly with $r$. The choice of scales and dimensionless parameters is always somewhat arbitrary, and different approaches can be found in the literature.

The evolution of flow $\mathbf{u}$ are described by the Navier-Stokes equation that formulates the momentum balance:

$$
E\left(\frac{\partial \mathbf{u}}{\partial t}+\mathbf{u} \cdot \nabla \mathbf{u}\right)+\mathbf{2} \hat{\mathbf{z}} \times \mathbf{u}+\nabla \Pi=\mathbf{E} \nabla^{\mathbf{2}} \mathbf{u}+\mathbf{R a} \frac{\mathbf{r}}{\mathbf{r}_{\mathbf{o}}} \mathbf{b}+\frac{\mathbf{1}}{P m}(\nabla \times \mathbf{B}) \times \mathbf{B} .
$$


The modified pressure $\Pi$ combines the nonhydrostatic pressure and centrifugal forces. Maxwell's laws and Ohm's law can be condensed into the induction equation:

$$
\frac{\partial \mathbf{B}}{\partial t}-\nabla \times(\mathbf{u} \times \mathbf{B})=\frac{\mathbf{1}}{\mathbf{P m}} \nabla^{2} \mathbf{B} .
$$

A simplified induction equation is solved in the conducting inner core, replacing flow $\mathbf{u}$ by the inner core rotation. Appropriate matching conditions guarantee the continuity of the magnetic field and the horizontal electric field at the inner core boundary (Wicht 2002).

Magnetic field and flow field are divergence free,

$$
\nabla \cdot \mathbf{B}=\mathbf{0}, \quad \nabla \cdot \mathbf{u}=\mathbf{0} .
$$

The latter condition is the simplified form of the continuity equation in the Boussinesq approximation, where density changes are retained only in the essential buoyancy term that drives convection in (2). This approximation is justified by the smallness of density variations in planetary iron cores.

Convection is driven by buoyancy effects resulting from two types of density variations. Freezing of the solid inner core releases light elements whose solubility is lower in solid than in liquid iron, sulfur is the most likely light constituent in the Hermian core (Breuer et al. 2007). Variations $\chi^{\prime}=\chi-\chi_{o}$ in the mean relative weight concentration of these light elements drive the so-called chemical or compositional convection. Here, $\chi_{o}=m_{S} /\left(m_{S}+\right.$ $\left.m_{\mathrm{Fe}}\right)$ is the homogeneous reference concentration in the outer core; $m_{S}$ and $m_{\mathrm{Fe}}$ are the total mass of sulfur, assumed to be the light constituent, and of iron, assumed to be the heavy constituent, respectively. Temperature variations, the driving source for thermal convection, have three principal origins: latent heat from inner core freezing, secular cooling, and heat production due to radiogenic elements. The simulations are concerned only with the superadiabatic temperature contribution $T^{\prime}$ that actually drives convective motions. Codensity $b$ combines thermal and chemical density variations in one variable:

$$
b=\alpha T^{\prime}+\beta \chi^{\prime} .
$$

Here, $\alpha$ is the thermal expansivity and $\beta$ is the equivalent chemical expansivity $\beta=$ $-\rho_{o}\left(\rho_{\mathrm{Fe}}-\rho_{S}\right) / \rho_{\mathrm{Fe}} \rho_{S}$, where $\rho_{\mathrm{Fe}}$ and $\rho_{S}$ are the densities of iron and sulfur in the outer core respectively (see also Sect. 4).

In principle, $T^{\prime}$ and $\chi^{\prime}$ obey two individual transport equations, which can be combined under the assumption that the effective (turbulent) thermal diffusivity $\kappa_{T}$ and compositional diffusivity $\kappa_{\chi}$ are identical (Braginsky and Roberts 1995; Kutzner and Christensen 2004):

$$
\frac{\partial b}{\partial t}+\mathbf{u} \cdot \nabla \mathbf{b}=\frac{\mathbf{1}}{\mathbf{P r}} \nabla^{\mathbf{2}} \mathbf{b}+\epsilon .
$$

The source/sink term $\epsilon$ represents various effects: possible radiogenic heat production, secular cooling, the destruction of compositional differences by mixing of the core fluid, and the loss of potential thermal buoyancy by the adiabatic gradient. On time average, $\epsilon$ must balance the codensity flux through the boundaries.

Different convective driving types can be modeled by choosing appropriate combinations of $\epsilon$ and outer and inner boundary conditions for the codensity variable $b$ (Kutzner and Christensen 2004). A simple and therefore common choice is to impose the codensity contrast $\Delta b$ over the simulated shell in combination with $\epsilon=0$. The inner boundary will then serve as a source, while the outer boundary forms the complementary sink. This approach 
was chosen for the thick-shell dynamos by Heimpel et al. (2005) as well as the thin-shell dynamos by Takahashi and Matsushima (2006) presented in Sect. 5.

Recent thermal evolution models suggest that the temperature gradient at the core-mantle boundary may actually be subadiabatic because the Hermian mantle developed a thick lithosphere early in the planets history, preventing the planet from cooling more efficiently. The core is unlikely to be completely solid for the same reason but has most likely cooled sufficiently to develop an inner core (Breuer et al. 2007). This leaves chemical convection as the main driving force for the dynamo. The subadiabatic thermal core-mantle boundary gradient translates into a negative codensity flux within the dynamo model outlined earlier $(\partial b / \partial r>0)$, i.e. both outer and inner boundaries serve as a source of $b$. We explore this interesting case in more detail in Sects. 4 and 6.

Appropriate flow and magnetic field boundary conditions close the system of differential equations ((2), (3), (4), (6)) that constitute the dynamo problem. So-called rigid flow boundary conditions are commonly employed, which implies that the radial flow component vanishes and that the fluid is corotating with the respective boundary. Stanley et al. (2005), however, assumed that the viscous shear stresses vanish at the boundaries, which defines an alternative condition for the horizontal flow components. This approach is motivated by the fact that viscous effects are much larger in the simulations than in planetary cores. Some authors (Zhang and Busse 1988; Kuang and Bloxham 1997) therefore have argued that neglecting the viscous boundary layers is dynamically more realistic than overestimating their effects.

Numerical dynamo simulations have the problem that they cannot run at realistic values of the control parameters. In particular, the kinematic viscosity has to be chosen several orders of magnitude too large in order to damp smaller scale turbulence that cannot be resolved numerically. As a result, Ekman and magnetic Prandtl numbers are significantly larger in the models than would be appropriate. A simple way to parameterize small-scale turbulent mixing is to assume larger effective diffusivities that are of comparable magnitude for all diffusive effects (Braginsky and Roberts 1995). This argument is commonly cited to justify the combination of thermal and compositional effects into one codensity variable and motivates the choice of $P r=1$ in numerical dynamo simulations. It does, however, not suffice to justify the significantly larger Ekman numbers required to compensate the inadequate numerical resolution. Table 1 lists parameter values for Mercury along with typical values assumed by the numerical simulations presented in the following. The value of the magnetic Reynolds number $R m=U D / \lambda$ is not known for Mercury since we cannot access the (RMS) core flow amplitude $U$. It is generally believed, however, that convective dynamos can only operate when $R m$ is large enough, say when $R m$ exceeds a critical value of 50 .

We refer to Takahashi et al. (2003) and Christensen and Wicht (2007) for details on the numerical methods.

Table 1 Parameter values for Mercury and for the dynamo simulations presented here. Only Christensen (2006) provides a $R o_{\ell}$ value for his dynamo model which is listed here

\begin{tabular}{lll}
\hline Parameter & Mercury & Simulations \\
\hline$E$ & $10^{-12}$ & $10^{-3}-10^{-5}$ \\
$P r$ (thermal) & 0.1 & 1 \\
$P r$ (compositional) & 100 & 1 \\
$P m$ & $10^{-6}$ & $1-5$ \\
$R m$ & $\geq 50$ & $100-1,000$ \\
$R o_{\ell}$ & 10 & 0.2 \\
\hline
\end{tabular}




\section{Estimating Planetary Magnetic Field Strengths}

Estimates of planetary magnetic field strengths are either based on the power available to drive the dynamo or are derived from force balances in the Navier-Stokes equation (2). We start with outlining the latter method.

\subsection{Mercury's Elsasser Number}

Dynamo theory distinguishes weak-field and strong-field cases. These adjectives relate to the role of the Lorentz force in the Navier-Stokes equation (2). Strong-field dynamos are characterized by a leading order force balance between Coriolis force, pressure gradient, and the Lorentz force. The Elsasser number $\Lambda$, which measures the ratio of Lorentz to Coriolis force, is therefore of order one in this so-called magnetostrophic regime:

$$
\Lambda=\frac{B^{2}}{\mu \lambda \rho_{o} \Omega} \approx 1 .
$$

With $B$ we denote the typical (RMS) magnetic field strength in the fluid shell.

Planetary dynamos are thought to operate in the strong-field regime and two arguments are commonly cited to justify this assumption. The first argument simply assumes that the Lorentz force has to enter the leading order balance to accomplish magnetic field saturation. Suppose that Lorentz forces were so weak that their back reaction on the fluid flow could be neglected. The dynamo equation can then be regarded as an eigenvalue problem for a given velocity field $\mathbf{u}$. The system is said to work as a kinematic dynamo when the set of eigen-solutions contains an exponentially growing mode. The amplitude of the respective eigen-vector will increase until the Lorentz force modifies the flow sufficiently to saturate the growth, which is supposedly the case when the Lorentz force enters the leading order force balance.

The second argument relies on the fact that a strong Lorentz force is needed to help convection by balancing the Coriolis force at least partly, thereby releasing the TaylorProudman constraint that severely restricts the flow vigor (Hollerbach 1996). The Lorentz force itself allows the flow to gain the strength necessary for the dynamo process and also increases the length scale of the flow, two effects that have been identified in dynamo simulations in Cartesian geometry (Rotvig and Jones 2002; Stellmach and Hansen 2004). However, these effects have not been found in the spherical-shell simulations that so successfully explain the geomagnetic field (Christensen and Aubert 2006), even though the Lorentz force indeed balances the Coriolis force to a good degree (Aubert 2005).

In order to access the Elsasser number $\Lambda$ of a planetary core we have to learn how to estimate the field strength in the dynamo region based on magnetic field measurements. Three different Elsasser numbers are introduced to discuss this issue: The classical Elsasser number $\Lambda$ is based on the RMS field strength in the dynamo regions, $\Lambda_{C M B}$ is the value calculated with the RMS field strength at the core-mantle boundary (CMB), and the dipole Elsasser number $\Lambda_{D}$ uses only the dipole contribution to the CMB field. The latter is assumed to be representative of the larger scale field, which is all we know in the case of Mercury. Including the relatively well-determined geomagnetic field as well as dynamo simulations into our discussion allows us to explore the different aspects of this problem.

Using recent geomagnetic field models for the year 2000 (Maus et al. 2006) we arrive at $\Lambda_{C M B} \approx 0.15$. Several issues prevent us from generalizing this value to represent core conditions. Earth's crustal magnetization contaminates any field contribution beyond spherical 
harmonic degree $l=14$. Consequently, these components are discarded in the internal field models, and their contribution to the Elsasser number is not known a priori. We also face the additional problem that there is no clear-cut way to extrapolate the poloidal magnetic field into the conducting dynamo region and, in addition, we lack any information on the toroidal field contribution.

Dynamo simulations provide the full magnetic field information and can therefore help to get an idea how $\Lambda_{C M B}$ and $\Lambda_{D}$ should be extrapolated to obtain the core value $\Lambda$. We first examine models that are geared to explain the geomagnetic field. These models are characterized by an inner core that occupies $35 \%$ of the total core radius and by convection that fills the whole outer core. A very Earth-like field geometry and amplitude can, for example, be found for $R a=18 \times R a_{c}$ ( $R a_{c}$ is the critical Rayleigh number for onset of convection), $E=3 \times 10^{-4}, P r=1$, and $P m=3$. This dynamo is representative of several nonreversing dipole-dominated cases examined by Christensen et al. (1999), Kutzner and Christensen (2004), and recently by Christensen and Aubert (2006). The time averaged CMB Elsasser number based on spherical harmonic degrees $l \leq 14$ is $\Lambda_{C M B} \approx 0.16$. The value increases to $\Lambda_{C M B} \approx 0.25$ when all spherical harmonic contribution are taken into account (the model is truncated at $l=85$ ). The RMS Elsasser number in the fluid shell is significantly larger, $\Lambda=5.16$, which suggests that the dynamo operates in the strong-field regime. About two thirds of the magnetic energy is contributed by the toroidal field. Examination of similar Earth-like dipole-dominated cases leads to the following rules of thumb: (a) including degrees beyond $l=14$ roughly doubles $\Lambda_{C M B}$; (b) the value based on the internal field strength is about an order of magnitude larger than the CMB value; and (c) toroidal and poloidal fields are of comparable amplitude. Translated to the geomagnetic field, this would suggest that Earth's Elsasser number is about $\Lambda=3$.

The dipole Elsasser number $\Lambda_{D}$, assumed to be representative of the coarse field information in the case of Mercury, amounts to about $\Lambda_{D}=0.05$ for recent geomagnetic field models and the dipole-dominated dynamo model mentioned earlier. When increasing the Rayleigh number in the simulations, the dynamo shows Earth-like reversal behavior with longer stable polarity epochs being interspersed by shorter excursions and reversals (Kutzner and Christensen 2002). We analyze a case at $R a=26 \times R a_{c}$. During stable polarity epochs, the dipole Elsasser number is somewhat lower than for the nonreversing case at $\Lambda_{D} \approx 10^{-2}$. It decreases significantly to $\Lambda_{D} \approx 3 \times 10^{-3}$ during excursion or reversals where the dipole loses its dominance (Kutzner and Christensen 2002; Christensen and Aubert 2006). We have averaged over several 10,000 years in both cases; $\Lambda_{D}$ can be somewhat lower or larger at times. The unstable-dipole period is also representative of the regime found at even higher Rayleigh numbers where a weak dipole constantly reverses and clear dipole-dominated stable epochs are missing altogether (Kutzner and Christensen 2002). The magnitude of roughly $\Lambda \approx 6$ in both the stable and the lowdipole epochs shows that the dynamo still operates in the strong field regime and demonstrates that the magnetostrophic balance can also be established by small scale magnetic field contributions.

Whether a dynamo operates in the dipole-dominated or in the constantly reversing regime where the dipole contribution is only marginal seems to be controlled by the local Rossby number, $R o_{\ell}=U /(\Omega \ell)$, where $\ell$ is a characteristic length scale of the flow (Christensen and Aubert 2006; Olson and Christensen 2006). The transitional value depends on various conditions, but is probably always less than 0.12 . Olson and Christensen (2006) suggested a scaling law that relates $R o_{\ell}$ to the fundamental control parameters and estimated a value $R o_{\ell} \approx 10$ for Mercury. This puts Mercury into the nondipolar regime, in apparent contradiction to the observed dominance of the axial dipole and quadrupole contributions. We come back to this point in Sect. 6 . 
Table 2 Physical parameters assumed for Mercury. Values have been taken from Spohn et al. (2001), Conzelmann (1999), and Braginsky and Roberts (1995)

\begin{tabular}{lll}
\hline Quantity & Symbol & Value \\
\hline Mean planetary radius & $R_{M}$ & $2,240 \mathrm{~km}$ \\
Core radius & $r_{o}$ & $1,850 \mathrm{~km}^{-6} \mathrm{sec}^{-1}$ \\
Rotation rate & $\Omega$ & $1.24 \times 10^{-1}$ \\
Mean mantle density & $\rho_{m}$ & $3,350 \mathrm{~kg} \mathrm{~m}^{-3}$ \\
Surface acceleration & $g_{M}$ & $3.8 \mathrm{~m} \mathrm{sec}^{-2}$ \\
Sulfur density & $\rho_{S}$ & $6,077 \mathrm{~kg} \mathrm{~m}^{-3}$ \\
Iron density & $\rho_{\mathrm{Fe}}$ & $8,412 \mathrm{~kg} \mathrm{~m}^{-3}$ \\
Thermal expansivity & $\alpha$ & $3 \times 10^{-5} \mathrm{~K}-1$ \\
Heat capacity & $c$ & $675{\mathrm{~J}\left(\mathrm{~kg} \mathrm{~K}^{-1}\right.}^{-1}$ \\
Latent heat & $L$ & $250 \times 10^{3} \mathrm{~W} \mathrm{~kg}^{-1}$ \\
Thermal diffusivity & $\kappa_{T}$ & $7 \times 10^{-6} \mathrm{~m}^{2} \mathrm{sec}^{-1}$ \\
Compositional diffusivity & $\kappa_{\chi}$ & $10^{-8} \mathrm{~m}^{2} \mathrm{sec}^{-1}$ \\
Kinematic viscosity & $\nu$ & $10^{-6} \mathrm{~m}^{2} \mathrm{sec}^{-1}$ \\
Electrical conductivity & $\sigma$ & $6 \times 10^{5} \mathrm{~S} \mathrm{~m}^{-1}$ \\
Magnetic permeability & $\mu$ & $4 \times 10^{-7} \pi \mathrm{N} \mathrm{A}^{-2}$ \\
\hline
\end{tabular}

Representing the Hermian field by a dipole contribution of $g_{10}=-325 \mathrm{nT}$ and assuming the properties listed in Table 2 we arrive at an Elsasser number of $\Lambda_{D} \approx 5 \times 10^{-5}$. This is more than two orders of magnitude lower than the lowest value $\left(\Lambda_{D} \approx 10^{-2}\right)$ in the Earthgeared dynamo simulations that still produce fields where the dipole contribution is stronger than the higher order components. To pose it differently, when using the extrapolations derived from geodynamo models we would limit the Elsasser number in Mercury's core to values less than $\Lambda \approx 10^{-2}$. This result could be interpreted in two ways: It could mean that Mercury's dynamo is not operating in the strong-field regime thought to be typical for planetary dynamos and found to be typical for geodynamo simulations. This conclusion motivated the development of the alternative dynamo models mentioned in the introduction. A second possibility is that the extrapolations fail because they are based on simulations that neglect important differences between Earth's and Mercury's interior. Potential candidates are the different relative size of Mercury's inner core and the particular type of convection owed to the fact that the core-mantle boundary heat flow is probably subadiabatic. These two issues are explored in Sects. 5 and 6, respectively.

\subsection{Dynamo Power Budget}

We proceed with exploring the dynamos power budget and related field strength estimates. Since the magnetic diffusivity generally exceeds the kinematic viscosity by several orders of magnitude, the energy dissipation is likely dominated by Ohmic effects in planetary dynamo regions. Balancing the energy input or power $P$ with Ohmic dissipation may then serve to estimate the field strength:

$$
P \approx \frac{\lambda V_{o} B^{2}}{\mu l_{\lambda}^{2}} .
$$

Here, we have introduced the length scale $l_{\lambda}$ at which the magnetic energy is supposedly dissipated most effectively (Christensen and Tilgner 2004), and we furthermore assume that Ohmic decay is mainly concentrated in the outer core volume $V_{o}$. Unfortunately, both power $P$ and diffusion length scale $l_{\lambda}$ are hard to constrain for planetary cores. 
Christensen and Tilgner (2004) explored Ohmic dissipation in a suit of dynamo models and suggested that magnetic energy is predominantly dissipated at a length scale

$$
l_{\lambda} \approx(1.74 / R m)^{1 / 2} D
$$

This result is supported by the fact that the Karlsruhe dynamo experiment seems to obey the same scaling (Christensen and Tilgner 2004). Equation (9) connects $l_{\lambda}$ with the convective vigor, a relationship that was investigated more closely by Christensen and Aubert (2006) based on an even larger suite of dynamo simulations than the one explored by Christensen and Tilgner (2004). Christensen and Aubert (2006) suggested that the flow amplitude is determined by the codensity flux $Q_{b, i}$ at the inner core boundary, and they proceeded to derive an empirical scaling that we also adopt here:

$$
R m \approx 0.83 \frac{P m}{E} R a_{b, i}{ }^{0.41} .
$$

The buoyancy or codensity flux Rayleigh number $R a_{b, i}$ is defined as:

$$
R a_{b, i}=\frac{1}{4 \pi r_{o} r_{i}} \frac{g_{o}}{\rho_{o} \Omega^{3} D^{2}} Q_{b, i}
$$

This scaling has been derived for dipole-dominated dynamos driven by vigorous convection that involves the whole fluid shell. Mercury's dynamo may actually fall into a different category, but the dependence of length scale $l_{\lambda}$ on the flow vigor and the dependence of the flow vigor on the codensity flux is certainly suggestive. The actual scaling factors and also, perhaps, the exponents may differ. By combining (7) with (8), (9), and (10) we arrive at a scaling that establishes the dependence of Elsasser number $\Lambda$ on codensity flux and power:

$$
\Lambda \approx 2 P m E^{2} P^{\prime} R a_{b, i}{ }^{-2 / 5} .
$$

The dimensionless power $P^{\prime}=P D^{4} /\left(V_{o} \rho_{o} v^{3}\right)$ has been rescaled using $\rho_{o} V_{o}$ as the mass scale. Christensen and Aubert (2006) pointed out that the buoyancy flux Rayleigh number is proportional to the nondimensional power, and they determined the factor of proportionality for the case when convection is driven by an imposed temperature contrast. The situation in Mercury's core is more complex because the combined action of thermal and compositional buoyancy may stabilize the outer part of the fluid core. In the following we derive the expression that links the buoyancy flux $Q_{b, i}$ to the heat flow at the core-mantle boundary and the relevant core properties. Furthermore, we determine the form factor $F$ that relates $P^{\prime}$ to $R a_{b, i}$, which will finally allow us to calculate the Elsasser number for a given buoyancy flux.

When estimating the total power available to drive the dynamo, we are interested in the long-term evolution of a mean reference state rather than the short-term convective fluctuations. Formally, this reference state represents a time-average over periods long enough to average out convective fluctuation but shorter than the relevant thermal and chemical evolution time scales. The laterally homogeneous reference state is characterized by a hydrostatic pressure and density profile $p(r)$ and $\rho(r)$, respectively. Furthermore, it is assumed that convection is strong enough to establish a chemically homogeneous and adiabatic reference state $T_{a}(r)$ in the lower convective part of the outer core (discussed later).

Compositional as well as thermal sources are available for driving the dynamo. Inner core growth and the associated release of light elements provide the compositional gravitational power $P_{C}$, which is subsequently converted to heat $Q_{C}=P_{C}$ by viscous effects. Additional (pseudo) heat sources are the change of internal energy due to secular cooling $Q_{S}$ and the 


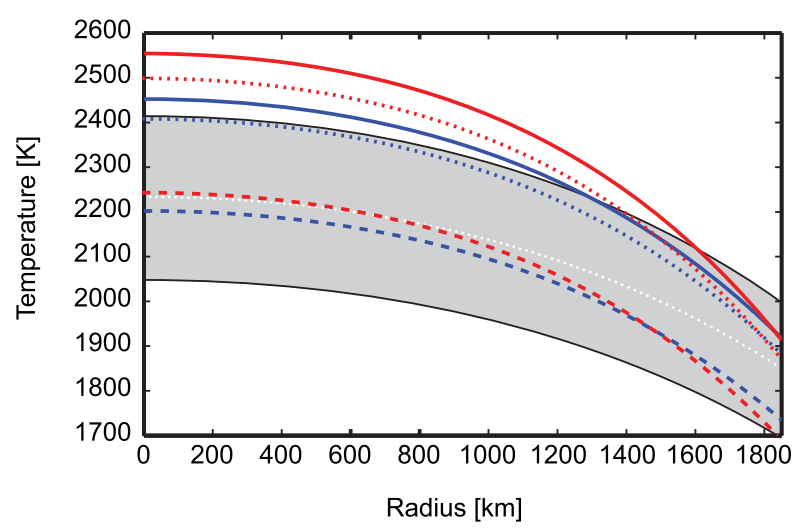

Fig. 5 Iron-sulfur melting curves assumed by Stevenson et al. (1983) (blue) and Hauk et al. (2004) (red), respectively. Values for sulfur weight fractions $\chi=0.001$ (solid), $\chi=0.01$ (dotted), and $\chi=0.05$ (dashed) are shown. The area between the core adiabats with core-mantle boundary temperatures of $T_{o}=1,700 \mathrm{~K}$ and $T_{o}=2,000 \mathrm{~K}$, respectively is marked in grey, white dots indicate the adiabat with $T_{o}=1,850 \mathrm{~K}$ (Stevenson et al. 1983; Schubert et al. 1988; Hauk et al. 2004)

release of latent heat $Q_{L}$ arising from inner core freezing. Ohmic heating and adiabatic heating have been neglected since they cancel when integrated over the core (Lister and Buffett 1995).

The total power available to the dynamo is given by:

$$
P=P_{C}+c_{i}\left(Q_{i}-Q_{A, i}\right)+c_{o}\left(Q_{o}-Q_{A, o}\right) .
$$

This expression assumes that the heat flux $Q_{i}=Q_{L}+Q_{S, i}$ coming in through the inner boundary $r_{i}$ is redistributed homogeneously throughout the outer core, so that the respective Carnot efficiency is given by $c_{i}=1-\bar{T} / T_{i} . Q_{S, i}$ represents effects of inner core secular cooling, $Q_{A, i}$ is the adiabatic heat flux through the inner boundary, $T_{i}$ is the temperature of the inner core surface, and $\bar{T}$ is the mass-averaged temperature (Lister and Buffett 1995). The superadiabatic heat flux $\left(Q_{o}-Q_{A, o}\right)$ at the outer boundary contributes with Carnot efficiency $c_{o}=\bar{T} / T_{o}-1$ to the available power; $Q_{o}=Q_{L}+Q_{S}+Q_{C}$ and $Q_{A, o}$ are the total and adiabatic heat flux at $r=r_{o}$, respectively.

The inner core radius is given by the crossing point of the core adiabat and the melting curve of the iron-sulfur mixture thought to be present in Mercury's core. However, neither the core adiabat nor the iron-sulfur melting curve are well determined. Figure 5 compares melting curves adopted by Stevenson et al. (1983) and Hauk et al. (2004) for three different core sulfur weight fractions: $\chi=0.001$ (solid), $\chi=0.01$ (dotted), and $\chi=0.05$ (dashed). The core sulfur content is largely unknown and depends on the distance at which the planetesimal formed that eventually built Mercury. Closer to the Sun less volatiles, including sulfur, would have been incorporated. Values from $\chi=0$ to $\chi=0.1$ have been considered by, for example, Hauk et al. (2004), Breuer et al. (2007).

Thermal evolution simulations (Stevenson et al. 1983; Schubert et al. 1988; Hauk et al. 2004; Breuer et al. 2007) suggest that Mercury's present core-mantle boundary temperature may lie between roughly $T_{o}=1,800 \mathrm{~K}$ and $T_{o}=2,000 \mathrm{~K}$. Some extreme models consider temperatures approaching $T_{o}=1,700 \mathrm{~K}$ (Hauk et al. 2004). Figure 5 shows the debated adiabatic range along with an example adiabat for $T_{o}=1,850 \mathrm{~K}$ and demonstrates how crucially $r_{i}$ depends on the melting-curve model, in particular for larger CMB temperatures. 
Sulfur contents beyond $\chi=0.05$ could mean that Mercury's core is still completely liquid, which is hard to reconcile with the convectively driven dynamo models considered here. We adopt the melting curve and adiabat parameterizations proposed by Stevenson et al. (1983) and, rather than depicting specific inner core radii, span the range $0<\eta<0.8$. Furthermore, we explore the three initial sulfur weight fractions $\chi_{0}=M_{S} /\left(M_{\mathrm{Fe}}+M_{S}\right)=0.001,0.01$, and 0.05 , where $M_{S}$ and $M_{\mathrm{Fe}}$ denote the total mass of sulfur and iron in the whole core, respectively. The sulfur weight fraction $\chi$ increases with growing inner core size: $\chi=\chi_{0} /\left(1-\eta^{3}\right)$.

The inner core radius changes in time due to the drop of the adiabat, caused by secular core cooling, and due to the drop of the melting curve with increasing $\chi$. These two effects establish the dependence of the rate of CMB temperature change $\dot{T}_{o}$ on the inner core growth rate $\dot{r}_{i}$ :

$$
\dot{T}_{o}=F_{T} \dot{r}_{i}
$$

The proportionality factor $F_{T}$ depends on $r_{i}$ and the adopted core model. Equation (14) allows us to write the heat change due to the secular-cooling $Q_{S}$ in terms of the inner core growth rate:

$$
Q_{S}=V_{c} \rho_{c} c \dot{T}_{o}=F_{S} \dot{r}_{i}
$$

For simplicity, we neglect the density difference between inner and outer core and use the mean core density $\rho_{c} ; V_{c}$ is the total core volume. Moreover, we assume that the inner core cools homogeneously.

The chemical power $P_{C}$ can be derived in two alternative ways: from calculating the change of the gravitational energy due to inner core growth (Schubert et al. 1988) or from considering the change of the reference state as described by the Navier-Stokes equation (2) and codensity equation (6) (Lister and Buffett 1995). Both methods lead to the same expression:

$$
P_{C}=6 \pi g_{o} \delta \rho \frac{\rho_{o}}{\rho_{\mathrm{Fe}}} r_{o}^{4}\left(\frac{1}{5}\left(1-\eta^{5}\right)-\frac{1}{3} \eta^{2}\left(1-\eta^{3}\right)\right) \frac{\dot{r}_{i}}{r_{i}}=F_{C} \dot{r}_{i},
$$

where $\delta \rho=\rho_{i}-\rho_{o}$ is the density difference between inner and outer core. We assume that the inner core contains no sulfur and that iron has the same density in the inner and outer core, so that the inner core density is $\rho_{i}=\rho_{\mathrm{Fe}}$. We furthermore neglect the small effects due to outer core shrinking (Schubert et al. 1988).

Using the dependence of inner core mass change $\dot{m}_{i}$ on the inner core growth rate,

$$
\dot{m}_{i}=4 \pi \rho_{i} r_{i}^{2} \dot{r}_{i}
$$

we can formulate the latent heat release $Q_{L}=L \dot{m}_{i}$ in terms of $\dot{r}_{i}$ :

$$
Q_{L}=3 L V_{i} \rho_{i} \frac{\dot{r}_{i}}{r_{i}}=F_{L} \dot{r}_{i},
$$

where $V_{i}$ is the inner core volume. Having established the dependence of all three heating/cooling contributions on $\dot{r}_{i}$ we compare the relative contributions to the heat budget, $Q_{C} / Q_{S}=F_{c} / F_{S}$ and $Q_{L} / Q_{S}=F_{L} / F_{S}$, in Fig. 6. The heat gain due to the conversion of chemical power is at least an order of magnitude smaller than the secular cooling effect, while latent heat and secular cooling effects are of comparable size. The difference between contributions due to chemical convection and due to latent heat release grows with increasing initial sulfur content $\chi_{0}$. 
Fig. 6 Contributions of compositional gravitational energy (black) and of latent heat (blue) to the heat flow budget relative to that of secular cooling. Sulfur weight fractions $\chi_{0}=0.001$ (solid), $\chi_{0}=0.01$ (dotted), and $\chi_{0}=0.05$ (dashed) have been considered

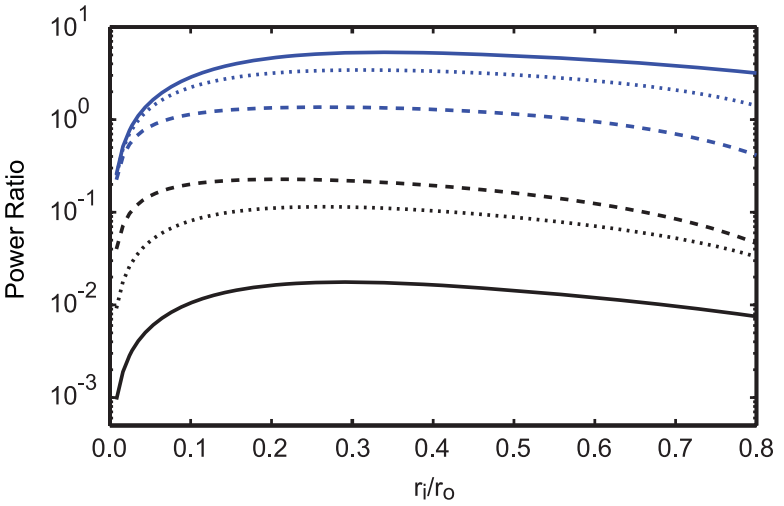

We can now derive the absolute inner core growth rate and the individual contributions to the CMB heat flux using (15), (16), and (18):

$$
\dot{r}_{i}=Q_{o} /\left(F_{C}+F_{L}+F_{S}\right) \approx Q_{o} /\left(F_{L}+F_{S}\right) .
$$

Above equations allow to derive the power avaible to drive Earth's dynamo where reasonable estimates of the $\mathrm{CMB}$ heat flux $Q_{o}$ and the inner core radius $r_{i}$ are available. For Mercury, where both quantities are unknown, we have to rely on thermal evolution models that couple mantle and core evolution via $Q_{o}$ and $T_{o}$ to form a combined model. Stevenson et al. (1983) and Hauk et al. (2004) parameterized the thermal evolution of both core and mantle; more recent simulations (Breuer et al. 2007) employ fully $3 \mathrm{D}$ mantle convection simulations. These models suggest that Mercury's CMB heat flow $Q_{o}$ may only have been super-adiabatic for a relatively brief period in the planet's history. The early formation of a thick lithosphere severely limits the heat flow, whose present day value may not exceed $5 \mathrm{~mW} / \mathrm{m}^{2}$. This is more than a factor two lower than the adiabatic value estimated to be about $12 \mathrm{~mW} / \mathrm{m}^{2}$ (Stevenson et al. 1983; Hauk et al. 2004), which has the unfortunate consequence that we cannot directly apply the power estimate (13).

A subadiabatic CMB heat flow suggests that the upper part of the core could be stably stratified, provided the stabilizing positive thermal buoyancy gradient exceeds the destabilizing negative compositional buoyancy gradient. The thermal gradient may change its sign to also become destabilizing deeper in the outer core. We have outlined in Sect. 3 how thermal and compositional effects can be combined into a codensity variable $b$. Formulating a power balance based on the codensity flux allows us to incorporate the effects of a subadiabatic outer boundary. We assume that convection is restricted to a region $r_{i} \leq r \leq r_{n}$, where $r_{n}$ is the neutral buoyancy radius $\partial \bar{b}\left(r_{n}\right) / \partial r=0$. The over-bar indicates an average over a spherical surface and over time. The relative smallness of the gravitational power allows us to neglect its contribution to the total CMB heat flux so that the inner core growth rate can be approximated by $\dot{r}_{i} \approx Q_{o} /\left(F_{L}+F_{S}\right)$. Given $\dot{r}_{i}$ we know $\dot{m}_{i}$ and can thus determine the inner core codensity or buoyancy flux:

$$
Q_{b, i}=\left(\frac{\rho_{\mathrm{Fe}}-\rho_{o}}{\rho_{\mathrm{Fe}}}+\frac{\alpha L}{c}\right) \dot{m}_{i}+\frac{\alpha}{c}\left(Q_{S, i}-Q_{A, i}\right) .
$$




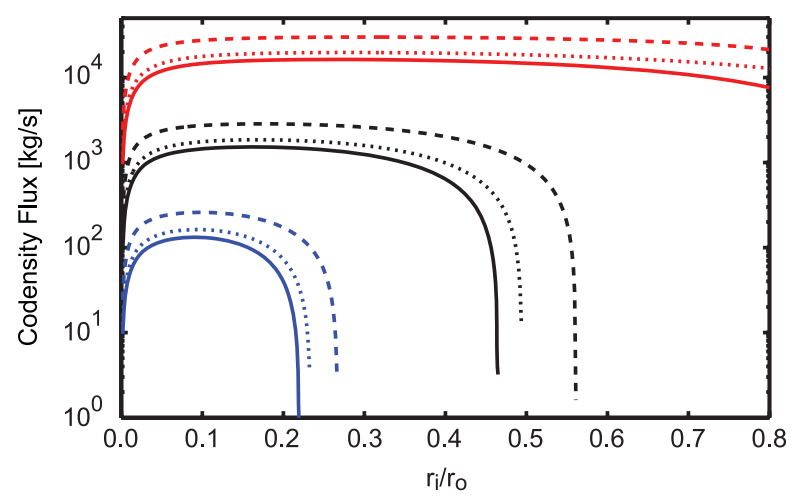

Fig. 7 Codensity or buoyancy flux at the inner boundary for the nine different models explored here. Cases for a CMB heat flux of $0.1 \mathrm{~mW} / \mathrm{m}^{2}, 1 \mathrm{~mW} / \mathrm{m}^{2}$, and $10 \mathrm{~mW} / \mathrm{m}^{2}$ are shown in blue, black, and red, respectively. The corresponding CMB buoyancy fluxes are $-3,822,-21,021$, and $-22,741 \mathrm{~kg} \mathrm{~s}^{-1}$ respectively. Solid, dotted, and dashed lines symbolize the inner codensity flux for initial sulfur weight fractions of $\chi_{0}=0.001$, $\chi_{0}=0.01$, and $\chi_{0}=0.05$ respectively

Fig. 8 Relative size of the convective zone $r_{i} \leq r \leq r_{n}$ for a core-mantle boundary heat flux of $0.1 \mathrm{~mW} / \mathrm{m}^{2}$ (blue), $1 \mathrm{~mW} / \mathrm{m}^{2}$ (black), and $10 \mathrm{~mW} / \mathrm{m}^{2}$ (red) and initial sulfur weight fractions of $\chi_{0}=0.001$ (solid), $\chi_{0}=0.01$ (dotted), and $\chi_{0}=0.05$ (dashed)

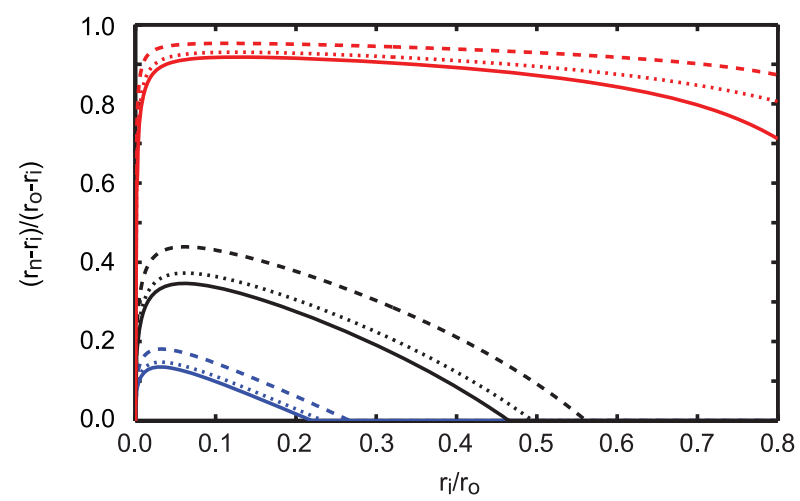

The codensity flux through the outer boundary is determined by the difference between the prescribed heat flow and the adiabatic heat flow:

$$
Q_{b, o}=\alpha / c\left(Q_{o}-Q_{A, o}\right) .
$$

Solving the time-averaged and horizontally averaged codensity diffusion equation, that describes the evolution of the reference state subject to the flux boundary conditions $Q_{b, o}$ and $Q_{b, i}$ then allows us to determine $r_{n}$. Figure 7 shows inner and outer codensity fluxes for CMB heat flows that cover three orders of magnitude, from mildly subadiabatic at $q_{o}=$ $10 \mathrm{~mW} / \mathrm{m}^{2}$ to heavily subadiabatic at $q_{o}=0.1 \mathrm{~mW} / \mathrm{m}^{2}$. The release of latent heat increases quadratically with inner core size $r_{i}$ (see (18)), while fixing the CMB heat flux limits the rate of latent heat release. Increasing the size of the inner core therefore leads to a decrease of the inner core growth rate, which in turn diminishes the codensity flux $Q_{b, i}$ that may ultimately become negative. A negative value of $Q_{b, i}$ means that the complete outer core is stably stratified leaving no convective energy to drive a dynamo.

Figure 8 shows the relative size of the convective zone, $\left(r_{n}-r_{i}\right) /\left(r_{o}-r_{i}\right)$, for the cases explored here. Assuming that Mercury's dynamo is indeed driven convectively we can ex- 
Fig. 9 Power available to drive the dynamo for the nine different cases explained in the caption of Fig. 7

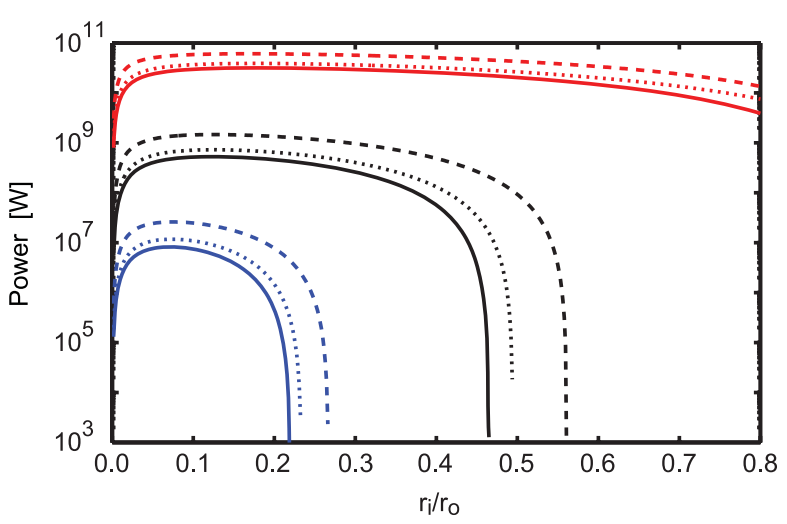

clude, for example, inner core radii beyond $55 \%$ of the total core radius when the CMB heat flux is only $1 \mathrm{~mW} / \mathrm{m}^{2}$ and beyond $25 \%$ when the flux is as low as $0.1 \mathrm{~mW} / \mathrm{m}^{2}$. The convective zone amounts to at most roughly $45 \%$ and $20 \%$ of the shell thickness, respectively, for these two cases.

The total power available to drive the dynamo can finally be calculated by modifying the chemical power expression (16) to also include the thermal buoyancy contribution,

$$
P=\frac{3}{2} \frac{g_{o}}{r_{o}} \frac{r_{n}^{2}}{1-\xi^{3}}\left(\frac{1}{5}\left(1-\xi^{5}\right)-\frac{1}{3} \xi^{2}\left(1-\xi^{3}\right)\right) Q_{b, i},
$$

with $\xi=r_{i} / r_{n}$.

Here we integrate over gravitational energy changes in the convective region $r_{i} \leq r \leq r_{n}$ but assume that $\rho_{o}$ is equivalent to a homogeneous distribution of light elements over the whole outer core. Figure 9 shows the dependence of the available power $P$ on aspect ratio $\eta=r_{i} / r_{o}$. For $q_{o}=1 \mathrm{~mW} / \mathrm{m}^{2}$, the power amounts to about $1 \mathrm{GW}$ for smaller inner cores and drops off rapidly for inner cores that fill more than $50 \%$ of the total core radius. For $q_{o}=0.1 \mathrm{~mW} / \mathrm{m}^{2}$, the power is roughly two orders of magnitude lower and drops off for relative inner core radii beyond $25 \%$. Ample power seems available for CMB fluxes of $q_{o}=10 \mathrm{~mW} / \mathrm{m}^{2}$ or larger.

The combination of (12) and (22) establishes the scaling of the Elsasser number $\Lambda$ with the inner boundary buoyancy-flux Rayleigh number (11):

$$
\Lambda \approx F P m E^{-1} R a_{b, i}{ }^{3 / 5}
$$

where $F$ is the form function:

$$
F=\frac{9}{5} \frac{\eta^{3}}{1-\eta^{3}} \frac{1-5 / 3 \xi^{2}+2 / 3 \xi^{5}}{\xi^{2}\left(1-\xi^{3}\right)} .
$$

To derive the Elsasser number in the convective region we replace $V_{o}$ in (12) with the appropriately modified volume $V_{n}$ of the convective region; Fig. 10 shows the respective results.

It is generally believed that convective dynamos cannot work at magnetic Reynolds numbers below, say, $R m=50$. This additional condition would limit the inner core radius to roughly $10 \%$ and $40 \%$ of the total core for $q_{o}=0.1 \mathrm{~mW} / \mathrm{m}^{2}$ and $q_{o}=1 \mathrm{~mW} / \mathrm{m}^{2}$, respectively. We have used (10) to calculate $R m$ here.

The Elsasser numbers are typically of order one or larger in all cases. Small values are only possible for very tiny inner cores, $r_{i} / r_{o}<0.01$, which is an unlikely scenario. Small 


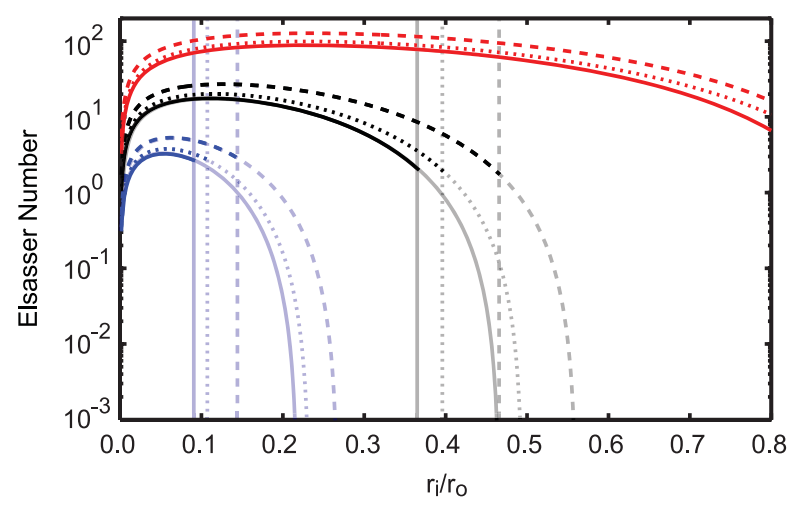

Fig. 10 Elsasser numbers for the power estimates in Fig. 9. Vertical lines mark aspect ratios beyond which the magnetic Reynolds number would fall below $R m=50$ for CMB heat flows of $0.1 \mathrm{~mW} / \mathrm{m}^{2}$ (blue) and $1 \mathrm{~mW} / \mathrm{m}^{2}$ (black), respectively. We switch to pale colors if $R m<50$. CMB heat flows of $10 \mathrm{~mW} / \mathrm{m}^{2}($ red $)$ are uncritical in this respect since $R m$ always exceeds the value $R m=50$ estimated to be a minimum requirement for convective dynamos

Elsasser numbers are also formally predicted for larger inner cores, close to the point where the codensity flux becomes negative. However, the magnetic Reynolds number is already too low for a self-sustained dynamo when the predicted $\Lambda$ drops below one.

In conclusion, the available power most likely suffices to drive a classical magnetostrophic planetary dynamo with an Elsasser number of order one or larger. This result is reassuring but not compliant with the measured magnetic field strength. Two effects can explain the differences: First, the Elsasser numbers given in Fig. 10 concern the RMS field strength in the convective region, which is more remote from the planetary surface than the CMB. Assuming that the magnetic field is dominated by the dipole contribution, an upward continuation to the CMB reduces the Elsasser number by about four orders of magnitude when the CMB heat flux is as low as $q_{o}=0.1 \mathrm{~mW} / \mathrm{m}^{2}$. In this particular case, the geometric effect is large enough to explain Mercury's low field strength because the dynamo is confined to a thin convective layer that surrounds a small inner core and is remote from the CMB. Thermal evolution simulations, however, suggest that the CMB heat flux is at least $q_{o}=1 \mathrm{~mW} / \mathrm{m}^{2}$ (Breuer et al. 2007). In this case, the geometric effect amounts to only two orders of magnitude, too small to bridge the gap between our Elsasser number estimates and the observed field strength. In Sect. 6 we will demonstrate that this goal will nevertheless be reached when we take into account that (a) Mercury's dynamo probably works in the nondipolar regime (discussed earlier) and that (b) the time-dependent magnetic field is attenuated by the electromagnetic skin-effect in the stratified conducting outer layer.

\section{Thin Shell and Thick Shell Dynamos}

We pointed out earlier that the size of Mercury's inner core is basically unknown. Several authors therefore explored how different inner core sizes would affect the dynamo process in models that were originally developed with the geodynamo in mind. A reduction of the externally observed field strength was found both for dynamos operating in a thin shell and dynamos operating in a thick shell, i.e. dynamos assuming rather small or rather larger inner cores. 
Thick shell dynamos were explored by Heimpel et al. (2005). They realized that the ratio $\Lambda_{C M B} / \Lambda$ decreases for smaller inner cores in their model. Although all their dynamos operate in the strong field regime, i.e $\Lambda \geq 1, \Lambda_{C M B}$ reaches values as low as $10^{-2}$ for $\eta=$ 0.15 which is the smallest aspect ratio they explored. This decrease can be traced to two causes: (1) the growing fraction of toroidal magnetic field produced by an $\omega$-effect, and (2) the local concentration of poloidal field generation. At $\eta=0.15$ radial convective motion is mainly concentrated in only one convective column that is also the center of poloidal magnetic field production. Consequently, the degree $m=1$ field component is somewhat pronounced. The toroidal field, on the other hand, is associated to a global zonal flow around the inner core. Roughly $75 \%$ of the magnetic energy at the CMB is carried by the dipole contribution, the field is thus still predominantly large scale and $\Lambda_{D}$ is not significantly smaller than $\Lambda_{C M B}$. The dipole tilt, judging from their Fig. 5a, seems compatible with the value $14 \pm 5^{\circ}$ inferred for Messenger data (Ness 1979). It should be mentioned that their simulations ran at only marginally supercritical Rayleigh numbers with respect to the onset of convection. A more supercritical convective motion will start to fill the whole shell and possibly lead to a different solution.

Simulations of dynamos that operate in thin shells have been carried out by Stanley et al. (2005) and Takahashi and Matsushima (2006). Stanley et al. (2005) explored aspect ratios between $\eta=0.7$ and $\eta=0.9$ and reported that thin-shell dynamos promote the production of a small-scale poloidal field as well as a strong axisymmetric toroidal magnetic field. While their dynamos operate in the strong-field regime, the observable dipole Elsasser number goes down to $\Lambda_{D}=10^{-3}$. The reason why the ratio $\Lambda_{D} / \Lambda$ lies below the value found by Heimpel et al. (2005) and the value discussed for the larger $R a$ cases presented in Sect. 4 is the dominant toroidal magnetic field in their simulations, which is up to an order of magnitude stronger than the poloidal field and establishes the magnetostrophic balance. As in the work of Heimpel et al. (2005), the low $\Lambda_{D}$ simulations presented by Stanley et al. (2005) operate at Rayleigh numbers close to the onset of dynamo action. At somewhat larger Rayleigh numbers the ratio of toroidal to poloidal field strength decreases and the Elsasser number $\Lambda_{D}$ increases. It should be noted that the neglect of viscous boundary layers (3) or the use of hyper-diffusivities seem to promote the stronger toroidal field (Stanley et al. 2005) not found in other dynamo simulations.

Takahashi and Matsushima (2006) also explored dynamos operating in a thin shell at $\eta=$ 0.7 and obtained dipolar as well as nondipolar dynamos. We present two examples here that ran at different Rayleigh numbers, $R a=3.50 \times R a_{c}$ (lower $R a$ case) and $R a=5.83 \times R a_{c}$ (larger $R a$ case), but otherwise identical parameters: $E=10^{-3}, \operatorname{Pm}=5$, and $\operatorname{Pr}=1$. The increase in Rayleigh number causes a change in the convective motion that goes along with a change in magnetic field geometries. A dipole dominated dynamo is found at the lower Rayleigh number, while the dipole has lost its dominance in the larger $R a$ case.

Figure 11(a) shows a snapshot of the radial magnetic field at the core surface in the lower Rayleigh number case. The field geometry is characterized by a longitudinal array of strong magnetic flux patches at mid-latitudes that corresponds to the outer rim of the tangent cylinder (TC) in both the northern and southern hemispheres. The tangent cylinder is an imaginary cylinder that is aligned with the rotation axis and touches the inner core equator. The field patches are created by flows converging where cyclonic convective columns connect with the CMB. These flows concentrate magnetic field lines and proceed down the columnar axis towards the equator (Olson et al. 1999). Because the convective columns are attached to the inner core, its size can possibly be inferred from the magnetic field morphology at the CMB. However, we note that, since the small-scale magnetic fields contributions decay more rapidly with distance from the source region, high-resolution measurements at satellite 

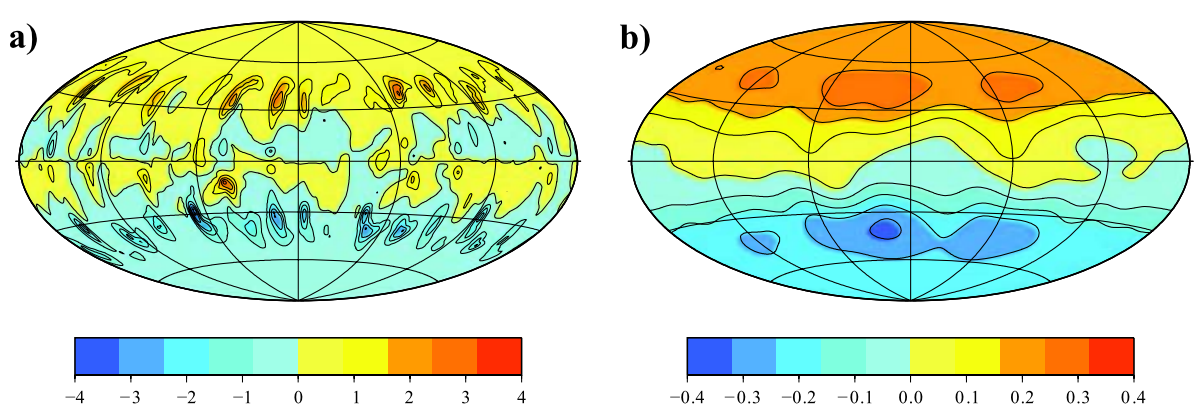

Fig. 11 The radial component of the magnetic field at (a) core surface and at (b) Mercury's surface level for $R a=3.50 \times R a_{c}$ in the thin-shell model by Takahashi and Matsushima (2006)
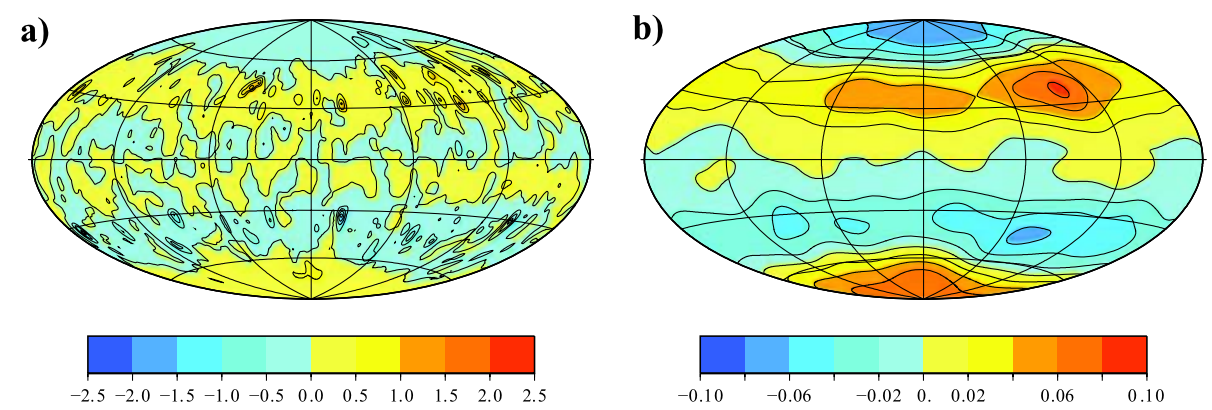

Fig. 12 Same as Fig. 11 but for the higher Rayleigh number case at $R a=5.83 \times R a_{c}$

altitude are required in order to discern these features. This is demonstrated by Fig. 11(b) where the now elongated flux patches are already much harder to identify at the planetary surface level (Takahashi and Matsushima 2006).

Figure 12 displays the magnetic field in the nondipolar case at a higher Rayleigh number. The structure exhibits several smaller strong flux patches distributed irregularly over the core surface. The increased field complexity is much less apparent at planetary surface level shown in Fig. 12(b). Such a complex magnetic field generally goes back to the increased spatial and temporal complexity of convective motion.

Columnar convective motion, which plays a key role in generating the magnetic field (Olson et al. 1999), occurs only outside the TC in thicker to intermediate shells. The region inside the TC is basically stagnant until plume-like convection starts at higher Rayleigh numbers. These localized upwellings typically create inverse magnetic field opposing the mean field direction outside the TC and tend to be highly time-dependent (Wicht and Olson 2004; Wicht 2005).

In a thin spherical shell, however, the region inside the TC occupies a much larger fraction, so that the localized and inverse field created by plumes rising at higher $R a$ has a bigger impact on the overall field geometry. Figure 12 clearly shows the large inverse field region inside the $\mathrm{TC}$ in both hemispheres, which are reflected in strong field components of octupolar and higher (odd) degrees. This is documented by the magnetic power spectra shown in Fig. 13. The magnetic power density $W_{l}$ contained in spherical harmonic degree $l$ 
Fig. 13 Magnetic power spectra with respect to spherical harmonic degree $l$ at the core surface (open symbols) and at planetary surface altitude (filled symbols) for dipolar (circles) and nondipolar (triangles) dynamos in the model by Takahashi and Matsushima (2006)

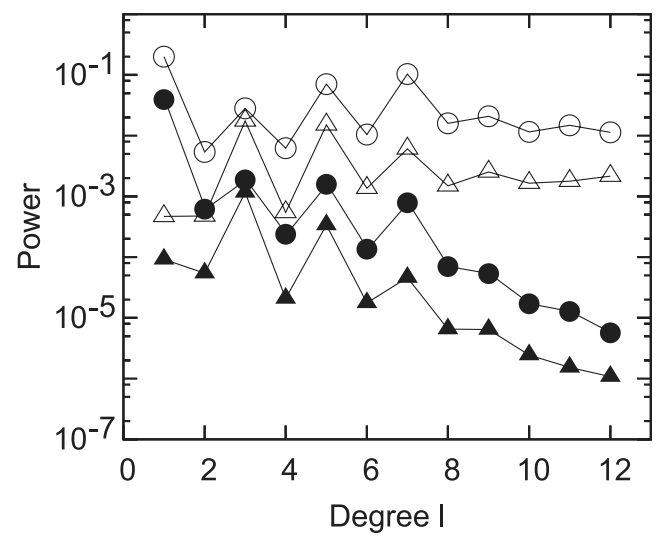

is defined as

$$
W_{l}(r)=(l+1)\left(\frac{r_{M}}{r}\right)^{2 l+4} \sum_{m=0}^{l}\left[\left(g_{l m}\right)^{2}+\left(h_{l m}\right)^{2}\right] .
$$

The spectrum of the nondipolar dynamo is characterized by nondipolar components at $l=3$ and $l=5$ at the core surface and by $l=3$ at the planetary surface. The axial octupole component actually dominates the field. Figure 13 also demonstrates that the field is generally smaller in the higher $R a$ case than for the dipole-dominated field (Takahashi and Matsushima 2005). Once again, the dynamo Elsasser number $\Lambda$ is of order one in the high $R a$ case, and the dipole Elsasser number is about $\Lambda_{D}=10^{-2}$.

While the models of Takahashi and Matsushima (2006) cannot ultimately explain the smallness of the observed field, they suggest that a precise measurement of Mercury's magnetic field may help to estimate the planet's inner core size, and thereby highlight the usefulness of global high-quality measurements.

\section{Dynamo in a Partly Stable Core}

Christensen (2006) addressed the question of how the presence of a stably stratified zone in the outer part of Mercury's core, as suggested by the subadiabatic CMB heat flow, would affect the dynamo process. Two dynamo models with different aspect ratios $\eta=0.35$ (case 1) and $\eta=0.50$ (case 2) have been explored. In both cases a nondimensional buoyancy flux of $\partial b / \partial r=-1$ is specified at the inner core boundary, but instead of prescribing the buoyancy flux at the CMB the volumetric sinks are set to $\epsilon=-1.235$ in case 1 and to $\epsilon=-1.5$ in case 2 . Boundary condition $b=0$ is enforced at the CMB. This approach allows the CMB buoyancy flux to vary in time, its mean values amount to -4 and -2.5 times the inner core boundary flux, respectively, and therefore fall within the range suggested by Fig. 7 for CMB heat flows of a few $\mathrm{mW} / \mathrm{m}^{2}$. The neutral buoyancy radius $r_{n}$ is such that only the lower $40 \%$ of the outer core are convectively unstable and the upper $60 \%$ are stable in both cases.

Since it is not possible to run dynamo models at the actual planetary values of the fundamental control parameters, care has been taken to ensure that the arguably most important nondimensional number, the magnetic Reynolds number, is in a realistic range for Mercury. The analysis by Christensen and Aubert (2006) suggests that $R m$ should obey scaling 

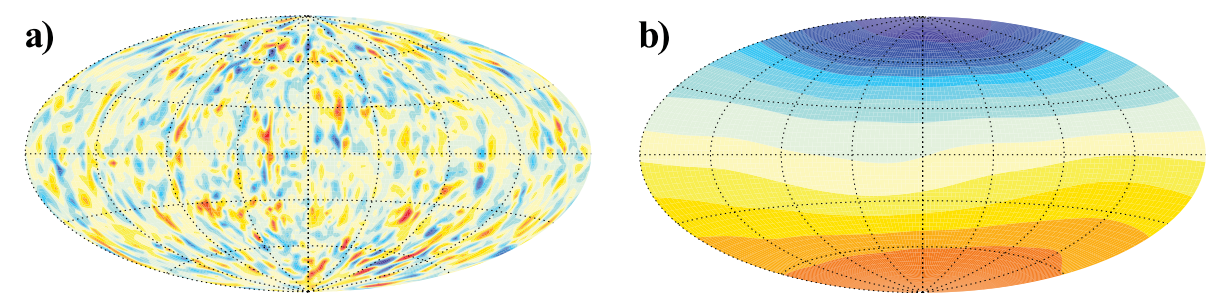

Fig. 14 Snapshot of the radial magnetic field in case 2. Panel (a): at radius $r=r_{i}+0.37 D$, i.e. near the top of the dynamo region with contour interval $40,000 \mathrm{nT}$; panel (b): at the planetary surface $\left(r=1.34 r_{o}\right)$ with contour interval $200 \mathrm{nT}$. Physical units of the magnetic field have been calculated assuming $\sigma=8 \times 10^{5} \mathrm{~S} \mathrm{~m}^{-1}$ and $\rho=8,200 \mathrm{~kg} \mathrm{~m}^{-3}$

law 10. The combination $\left(P m E^{-1} R a_{b i}{ }^{0.41}\right)$ is of order 1,000 for the Hermian parameter values assumed here, and the same value is obtained for the chosen model parameters: $\left(R a_{b}, E, P m, P r\right)=\left(1.08 \times 10^{-5}, 3 \times 10^{-5}, 3,1\right)$ in case 1 and $\left(2 \times 10^{-4}, 10^{-4}, 3,1\right)$ in case 2 . The model value of the magnetic Reynolds number should thus match the planetary value. Another important consideration is that the models should be in a regime with a sufficiently large value of the local Rossby number $R o_{\ell}$ to make sure that the dynamo operates in the nondipole dominated regime. Although the value appropriate for Mercury, $R o_{\ell} \approx 10$, cannot be reached in the numerical simulations, $R o_{\ell}$ is large enough to ensure that the dynamo operates in the correct regime (Olson and Christensen 2006).

Though active convection is restricted to the unstable lower part of the outer core, the circulation nevertheless penetrates into the upper region in the form of Taylor columns aligned with the rotation axis. The respective flow is almost entirely horizontal (toroidal) and weaker than in the convection region (Christensen 2006). Figure 14 shows a snapshot of the radial magnetic field for model case 2. As expected, the field is strong and small-scaled in the dynamo region without a clearly discernable dipole component (upper panel). However, the field is dominated by an axial dipole component at the planetary surface where the field intensity is considerably weaker. While the core Elsasser number is approximately $\Lambda=2.5$, the CMB value is significantly smaller at $\Lambda_{C M B}=2 \times 10^{-4}$. This is remarkably close to the observed value, which is about twice as small.

The strong dipole dominance of the field outside the core is also obvious in the magnetic power spectrum (see Fig. 15(b), circles), which decays rapidly with increasing harmonic degree $l$. In contrast, the spectrum inside the core (crosses) peaks at degree 4 , where the energy is four times larger than at $l=1$. The spectrum is rather flat out to $l=30$. In case 1 , the power spectrum inside the core is similar to that of case 2 (Fig. 15(a)). The surface spectrum again shows a rapid decay for $l>3$, but the dipole contribution is weaker than in case 2 . On time average, dipole and quadrupole components are of comparable strength, but one or the other may dominate at some instance in time (Christensen 2006). The axisymmetric components are usually more prominent than nonaxisymmetric components in the outside field. They clearly dominate in case 2 and, on average, carry about $2 / 3$ of the energy in case 1 . In contrast, they contribute only $6 \%$ to the poloidal magnetic energy inside the dynamo region. The average surface field is about a factor five weaker than the observed field in case 1 .

One reason for the differences in spectral power between the field in the dynamo region and the surface field is the geometric decrease with radius, according to $r^{-(l+2)}$ for a potential field. However, the reduction in amplitude is larger than that of simple geometric decay for all field components in case 1 and for all components except the axial dipole in case 2 . The dynamo-generated magnetic field must essentially diffuse through the stable conducting 

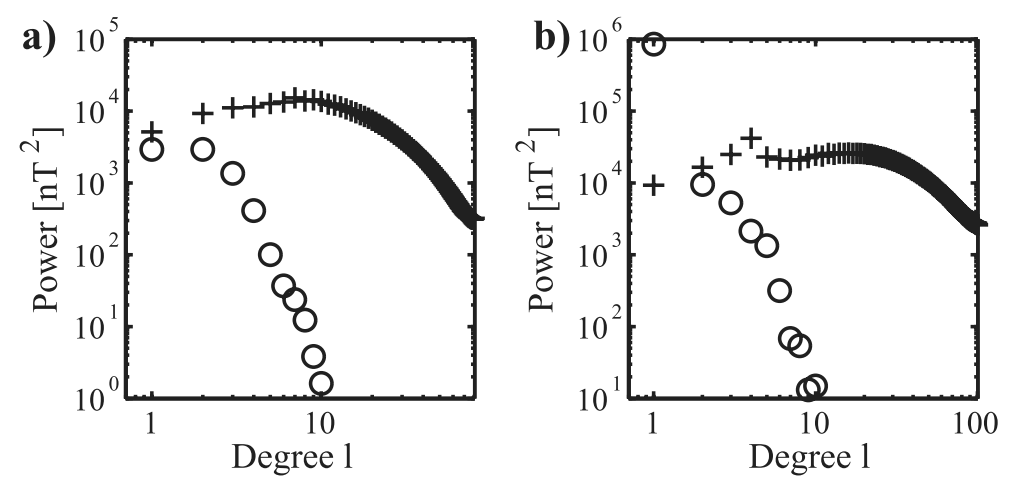

Fig. 15 Time-average magnetic power spectra versus harmonic degree $n$ for (a) case 1 and (b) case 2 . Circles refer to the planetary surface and crosses to the mean energy inside the fluid core, scaled down by a factor $10^{-4}$
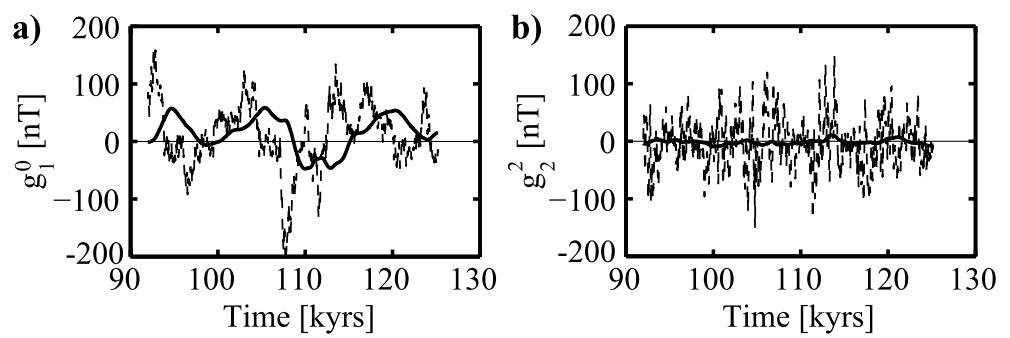

Fig. 16 Axial dipole coefficient (a) and coefficient for a nonaxial quadrupole component (b) versus time for case 1. Full lines: Gauss coefficient at Mercury's surface; broken lines: equivalent coefficients for the poloidal field at $r=r_{i}+D / 2$, scaled down by a factor 0.1

layer, ignoring for simplicity induction effects by the horizontal flow in this layer. The field is time-dependent and attenuated by the skin effect.

Figure 16(a) compares the time variation of the axial dipole field slightly above the dynamo region and at the planetary surface for case 1 . Rapid fluctuations are completely eliminated, whereas slow variations on a 10,000 yrs time scale penetrate to a limited degree. At the surface they are phase-shifted by about 2,500 yrs, as expected for the skin-effect. Nonaxisymmetric components of the dynamo field and, in general, higher multipole components $l>2$ fluctuate rapidly on short time-scales (Fig. 16(b)). Though these contributions dominate in the dynamo region, they are strongly suppressed in the surface field. The characteristic time scale of magnetic field changes in dynamos has been found to vary with the inverse magnetic Reynolds number (Christensen and Tilgner 2004). Since $R m$ is in the correct range in the models the time-scales should also be about correct.

In case 2, the dipole field is much more stable and, in contrast to case 1 , does not reverse during the 86,000 yrs worth of simulation. Since the DC part of the dipole field is not affected by the skin effect, case 2 retains a comparatively strong dipole outside the core. The enhanced dipole stability could be a consequence of the increased magnetic inertia represented by the larger solid inner core (Hollerbach and Jones 1993; Takahashi and Matsushima 2006), which might prevent short-term fluctuations of the dipole in the dynamo region from growing into complete polarity reversals. 
Because of the filtering effect of the stably stratified layer secular-variation, time scales of the externally observable field are much longer than the intrinsic time scales of the dynamo process. Significant variations of Mercury's field can thus only be expected on time scales of centuries or longer. Both the dipolar field geometry of case 2 and the strong axial quadrupole contribution that exists at some times in case 1 are compatible with the limited knowledge about Mercury's field structure (Connerney and Ness 1988). Although the surface field is too weak in case 1 and too strong in case 2, moderate changes such as decreasing the thickness of the stable layer in case 1 or driving convection more strongly to force the dipole to reverse in case 2 could bring the field strength into line with the observed intensity.

\section{Conclusion}

Dynamo theory suggests that convective planetary dynamos operate in the strong-field regime where Lorentz forces and Coriolis forces are of comparable magnitude and establish the so-called magnetostrophic balance. In other words, the Elsasser number $\Lambda$, the ratio of Lorentz to Coriolis forces, should be of order one. While this seems to be true for Earth, Jupiter, Saturn, and possibly also the ice giants (Stevenson et al. 1983), Mercury violates this rule on first sight. An extrapolation of the weak field measured by Mariner 10 to the dynamo region suggests that $\Lambda$ is as low as $10^{-4}$.

Alternative explanations therefore promote, for example, crustal magnetization (Aharonson et al. 2004) or a thermoelectric mechanism (Stevenson 1987; Giampieri and Balogh 2002). We have reviewed several convective dynamo simulations, which suggest that the discrepancy may come about because the observed surface field does not reflect the true field strength within the core. Two possible reasons for this are: (1) the magnetostrophic balance is established to a substantial degree by small-scale magnetic field that has not been resolved by the observations, and (2) the balance is mainly maintained by the toroidal field, which cannot be detected outside the core. Significant small-scale contributions and/or strong toroidal fields are promoted by either particularly small inner cores (thick shell dynamo) (Heimpel et al. 2005) but also by large inner cores (thin shell dynamo) (Stanley et al. 2005; Takahashi and Matsushima 2006). The discrepancy between the Elsasser number $\Lambda_{D}$, based on the observable large scale dipole field, and $\Lambda$, based on the RMS field in the dynamo region, can reach three orders of magnitude (Stanley et al. 2005).

Analyses by Christensen and Aubert (2006) and Olson and Christensen (2006) suggest that a parameter called the local Rossby number $R o_{\ell}$ decides whether a dynamo falls into one of two distinct families. Dipole-dominated fields are produced for small local Rossby numbers but the dipole loses its dominance when $R o_{\ell}$ is increased beyond about 0.12 . The value $R o_{\ell} \approx 10$ estimated for Mercury puts the planet into the second category. Analyses of a dynamo operating in this second regime, but with an Earth-like relative inner-core size, shows that the dynamo Elsasser number is still of order one but that the large scale dipole value $\Lambda_{D}$ is nearly three orders of magnitude lower.

Thin-shell, thick-shell, and also high- $R o_{\ell}$ dynamos produce magnetic fields with significant small-scale contributions. These dynamos can possibly be ruled out should Messenger and BepiColombo measurements confirm the impression that Mercury's field is dominated by large-scale components, i.e. dipole and quadrupole. Should the measurements, on the other hand, resolve smaller scale field patches, they may help to constrain the size of Mercury's inner core, since these features tend to coalesce at the tangent cylinder that touches the inner-core equator.

Christensen (2006) explored a somewhat different dynamo model that is motivated by estimates of the core power budget. Thermal evolution simulations suggest that the heat flow 
at the planet's core-mantle boundary may be subadiabatic. The available power nevertheless suffices to drive a magnetostrophic dynamo $(\Lambda=1)$ due to chemical differentiation, latent heat release, and secular cooling. However, a subadiabatic CMB heat flow has the consequence that the outer part of the core may be stably stratified. Christensen (2006) simulated cases where only the lower $40 \%$ of the core convect actively; he also made sure that $R o_{\ell}$ is large enough for the dynamo to operate in the nondipole-dominated regime. Key points of this model are: (1) the field in the dynamo region is strong but is dominated by small scales with rather weak dipole and quadrupole contributions, (2) the field is time-dependent with small-scale components varying more rapidly than low-order components, and (3) the dynamo field must diffuse through the stable conducting region. Here the rapidly varying high-order components are filtered out by the skin effect, whereas the slowly varying dipole and quadrupole components pass with some attenuation.

While the resulting field obeys $\Lambda \approx 1$ in the dynamo region, its surface value is close to the measured amplitude, so that these models successfully explain the planet's measured low field strength (Christensen 2006). Two other magnetic field features seem to distinguish the models by Christensen (2006) and those that do not incorporate a stagnant outer-core region (Stanley et al. 2005; Heimpel et al. 2005; Takahashi and Matsushima 2006). First, the smaller scale field contributions are significantly less pronounced, and second, secular variation takes place on time scales of some centuries rather than decades. Thus the model with a partly stagnant fluid core would be supported should Messenger and BepiColombo find little magnetic field change compared to the Mariner 10 epoch and also confirm that the field is indeed predominantly large scale.

\section{References}

M.H. Acuña, J.E.P. Connerney, N.F. Ness, R.P. Lin, D. Mitchell, C.W. Carlson, J. McFadden, K.A. Anderson, H. Réme, C. Mazelle, D. Vignes, P. Wasilewski, P. Cloutier, Global distribution of crustal magnetization discovered by the Mars Global Surveyor MAG/ER experiment. Science 284, 790-793 (1999)

O. Aharonson, M.T. Zuber, S. Solomon, Crustal remanence in an internally magnetized non-uniform shell: A possible source for Mercury's magnetic field? Earth Planet. Sci. Lett. 218, 261-268 (2004)

K.A. Anderson, D.E. Wilhelms, Correlation of lunar farside magnetized regions with ringed impact basins. Earth Planet. Sci. Lett. 46, 107-112 (1979)

J. Arkani-Hamed, Magnetization of Martian lower crust: Revisited. J. Geophys. Res. 112(E11), 5008 (2007)

N. Artemieva, L. Hood, B. Ivanov, Impact demagnetization of the Martian crust: Primaries versus secondaries. Geophys. Res. Lett. 32, L22204 (2005)

J. Aubert, Steady zonal flows in spherical shell dynamos. J. Fluid Mech. 542, 53-67 (2005)

S.I. Braginsky, P.H. Roberts, Equations governing convection in Earth's core and the geodynamo. Geophys. Astrophys. Fluid Dyn. 79, 1-97 (1995)

D. Breuer et al., Interior evolution of Mercury. Space Sci. Rev. (2007). doi:10.1007/s11214-007-9228-9

D. Breuer, T. Spohn, Early plate tectonics versus single-plate tectonics on Mars: Evidence from magnetic field history and crust evolution. J. Geophys. Res. (Planets) 108, 8-1 (2003)

M. Buske, U.R. Christensen, (2007). Three-dimensional convection models for the thermal evolution of the martian interior (2007, in prep.)

L. Carporzen, S. Gilder, R. Hart, Palaeomagnetism of the Vredefort meteorite crater and implications for craters on Mars. Nature 435, 198-201 (2005)

U. Christensen, J. Wicht, Numerical dynamo simulations, in Core Dynamics, Treatise on Geophysics (Elsevier, 2007)

U. Christensen, P. Olson, G.A. Glatzmaier, Numerical modeling of the geodynamo: A systematic parameter study. Geophys. J. Int. 138, 393-409 (1999)

U.R. Christensen, A deep rooted dynamo for Mercury. Nature 444, 1056-1058 (2006)

U.R. Christensen, J. Aubert, Scaling properties of convection-driven dynamos in rotating spherical shells and applications to planetary magnetic fields. Geophys. J. Int. 166, 97-114 (2006)

U.R. Christensen, A. Tilgner, Power requirement of the geodynamo from ohmic losses in numerical and laboratory dynamos. Nature 429, 169-171 (2004) 
U.R. Christensen, J. Aubert, F.H. Busse, P. Cardin, E. Dormy, S. Gibbons, G.A. Glatzmaier, Y. Honkura, C.A. Jones, M. Kono, M. Matsushima, A. Sakuraba, F. Takahashi, A. Tilgner, J. Wicht, K. Zhang, A numerical dynamo benchmark. Phys. Earth Planet. Interiors 128, 25-34 (2001)

J.E.P. Connerney, N.F. Ness, Magnetic field and interior, in Mercury, ed. by F. Vilas, C.R. Chapman, M.S. Matthews (The University of Arizona Press, Tucson, 1988), pp. 494-513

J.E.P. Connerney, M.H. Acuña, N.F. Ness, G. Kletetschka, D.L. Mitchell, R.P. Lin, H. Rme, Tectonic implications of Mars crustal magnetism. Proc. Nat. Acad. Sci. 102, 42 (2005)

V. Conzelmann, Thermische Evolutionsmodelle des Planeten Merkur berechnet unter der Anwendung verschiedener Viskositätsgesetzte. Ph.D. thesis, Westfälische Wilhelms Universität Münster (1999)

M. Fujimoto, W. Baumjohann, K. Kabin, R. Nakamura, J.A. Slavin, N. Terada, L. Zelenyi, Space Sci. Rev. (2007, this issue). doi:10.1007/s11214-007-9245-8

G. Giampieri, A. Balogh, Mercury's thermoelectric dynamo model resvisisted. Planet. Space Sci. 50, 757762 (2002)

Glassmeier, K.-H., Grosser, J., Auster, H.-U., Constantinescu, D., Narita, Y., Stellmach, S., Electromagnetic induction effects and dynamo action in the Hermean system. Space Sci. Rev. (2007). doi:10.1007/ s11214-007-9244-9

S.A. Hauk, A.J. Dombard, R.J. Phillips, S.C. Solomon, Internal and tectonic evolution of Mercury. Earth Planet. Sci. Lett. 222, 713-728 (2004)

M.H. Heimpel, J.M. Aurnou, F.M. Al-Shamali, N. Gomez Perez, A numerical study of dynamo action as a function of sperical shell geometry. Phys. Earth Planet. Interiors 236, 542-557 (2005)

R. Hollerbach, On the theory of the geodynamo. Phys. Earth Planet. Interiors 98, 163-185 (1996)

R. Hollerbach, C.A. Jones, Influence of the Earth's inner core on geomagnetic fluctuations and reversals. Nature 365, 541-543 (1993)

L.L. Hood, A. Zakharian, J. Halekas, D.L. Mitchell, R.P. Lin, M. Acuña, A.B. Binder, Initial mapping and interpretation of lunar crustal magnetic anomalies using Lunar Prospector magnetometer data. J. Geophys. Res. 106, 27,825-27,839 (2001)

W. Kuang, J. Bloxham, An Earth-like numerical dynamo model. Nature 389, 371-374 (1997)

C. Kutzner, U.R. Christensen, From stable dipolar to reversing numerical dynamos. Phys. Earth Planet. Interiors 131, 29-45 (2002)

C. Kutzner, U.R. Christensen, Simulated geomagnetic reversals and preferred virtual geomagnetic pole paths. Geophys. J. Int. 157, 1105-1118 (2004)

B. Langlais, M.E. Purucker, M. Mandea, The crustal magnetic field of Mars. J. Geophys. Res. 109 (2004).

V. Lesur, S. Maus, A global lithospheric magnetic field model with reduced noise level in the polar regions. Geophys. Res. Lett. 33 (2006)

J.R. Lister, B.A. Buffett, The strength and efficiency of thermal and compositional convection $\mathrm{n}$ the geodynamo. Phys. Earth Planet. Interiors 91, 17-30 (1995)

J.G. Luhmann, The solar wind interaction with Venus. Space Sci. Rev. 44, 241 (1986)

M. Mandea, M. Purucker, Observing, modeling, and interpreting magnetic fields of the solid Earth. Surv. Geophys. 26, 415-459 (2005)

M. Mandea, E. Bellanger, J.-L. Le Mouël, A geomagnetic jerk for the end of 20th century? Earth Planet. Sci. Lett. 183, 369-373 (2000)

J.L. Margot, S.J. Peale, R.F. Jurgens, M.A. Slade, I.V. Holin, Large longitude libration of Mercury reveals a molten core. Science 316, 710-00 (2007)

S. Maus, M. Rother, C. Stolle, W. Mai, S. Choi, H. Lühr, D. Cooke, C. Roth, Third generation of the potsdam magnetic model of the earth (POMME). Geochem. Geophys. Geosyst. 7, 7008 (2006)

N.F. Ness, The magnetic field of Mercury. Phys. Earth Planet. Interiors 20, 209-217 (1979)

N. Olsen, H. Lühr, T.J. Sabaka, M. Mandea, M. Rother, L. Tofner-Clausen, S. Choi, Geophys. J. Int. 166, 67-75 (2006)

P. Olson, U.R. Christensen, Dipole moment scaling for convection-driven planetary dynamos. Earth Planet. Sci. Lett. 250, 561-571 (2006)

P. Olson, U. Christensen, G.A. Glatzmaier, Numerical modeling of the geodynamo: Mechanism of field generation and equilibration. J. Geophys. Res. 104, 10,383-10,404 (1999)

J. Rotvig, C.A. Jones, Rotating convection-driven dynamos at low Ekman number. Phys. Rev. E 66(5), 056308 (2002)

S.K. Runcorn, An acient lunar magnetic dipole field. Nature 253, 701-703 (1975)

G. Schubert, M.N. Ross, D.J. Stevenson, T. Spohn, Mercury's thermal history and the generation of its magnetic field, in Mercury, ed. by F. Vilas, C.R. Chapman, M.S. Matthews (The University of Arizona Press, Tucson, 1988), pp. 651-666

G. Siscoe, N.F. Ness, C.M. Yeates, Substorms on Mercury? J. Geophys. Res. 80, 4359 (1975)

T. Spohn, M.H. Acuña, D. Breuer, M. Golombek, R. Greeley, A. Halliday, E. Hauber, R. Jaumann, F. Sohl, Geophysical constraints on the evolution of Mars. Space Sci. Rev. 96, 231-262 (2001) 
T. Spohn, F. Sohn, K. Wieczerkowski, V. Conzelmann, The interior structure of Mercury: What we know, what we expect from BepiColombo. Planet. Space Sci. 49, 1561-1570 (2001)

S. Stanley, J. Bloxham, W.E. Hutchison, M.T. Zuber, Thin shell dynamo models consistent with Mercury's weak observed magnetic field. Earth Planet. Sci. Lett. 234, 341-353 (2005)

S. Stellmach, U. Hansen, Cartesian convection driven dynamos at low Ekman number. Phys. Rev. E 70(5), $056312(2004)$

D.J. Stevenson, Mercury's magnetic field: A thermoelectric dynamo? Earth Planet. Sci. Lett. 82, 114-120 (1987)

D.J. Stevenson, T. Spohn, G. Schubert, Magnetism and thermal evolution of terrestrial planets. Icarus 54, 466-489 (1983)

F. Takahashi, M. Matsushima, Dynamo action in a rotating spherical shell at high Rayleigh numbers. Phys. Fluids 17, 076601 (2005)

F. Takahashi, M. Matsushima, Dipolar and non-dipolar dynamos in a thin shell geometry with implications for the magnetic field of Mercury. Geophys. Res. Lett. 33, L10202 (2006)

F. Takahashi, M. Matsushima, Y. Honkura, Dynamo action and its temporal variation inside the tangent cylinder in MHD dynamo simulations. Phys. Earth Planet. Interiors 140, 53-71 (2003)

R. Tyler, L. Mysak, J. Oberhuber, Electromagnetic fields generated by a three dimensional global ocean circulation. J. Geophys. Res. 102, 5531-5551 (1997)

R. Tyler, A. Maus, H. Lühr, Satellite observations of magnetic fields due to ocean tidal flow. Science 299, 239-241 (2003)

B.P. Weiss, S.S. Kim, J.L. Kirschvink, R.E. Kopp, M. Sankaran, A. Kobayashi, A. Komeili, Ferromagnetic resonance and low-temperature magnetic tests for biogenic magnetite. Earth Planet. Sci. Lett. 224, 73 89 (2004)

J. Wicht, Inner-core conductivity in numerical dynamo simulations. Phys. Earth Planet. Interiors 132, 281 $302(2002)$

J. Wicht, Palaeomagnetic interpretation of dynamo simulations. Geophys. J. Int. 162, 371-380 (2005)

J. Wicht, P. Olson, A detailed study of the polarity reversal mechanism in a numerical dynamo model. Geochem. Geophys. Geosyst. 5, 3 (2004)

J.-P. Williams, F. Nimmo, Thermal evolution of the Martian core: Implications for an early dynamo. Geology 32, 97 (2004)

K.-K. Zhang, F.H. Busse, Finite amplitude convection and magnetic field generation in in a rotating spherical shell. Geophys. Astrophys. Fluid Dyn. 44, 33-53 (1988) 